\title{
Berechnung von Temperaturfahnen im Grundwasser mit analytischen und numerischen Modellen
}

\author{
Marc Ohmer $^{1}$ (D) $\cdot$ Artur Klester $^{1} \cdot$ Alexander Kissinger $^{2} \cdot$ Stefan Mirbach ${ }^{2} \cdot$ Holger Class $^{3} \cdot$ Martin Schneider $^{3}$. \\ Martin Lindenlaub ${ }^{4} \cdot$ Michael Bauer $^{5} \cdot$ Tanja Liesch $^{1} \cdot$ Kathrin Menberg $^{1} \cdot$ Philipp Blum ${ }^{1}$
}

Eingegangen: 8. Juli 2021 / Überarbeitet: 29. November 2021 / Angenommen: 8. Dezember 2021 / Online publiziert: 7. März 2022

๑) Der/die Autor(en) 2022, korrigierte Publikation 2022

\section{Zusammenfassung}

Mit zunehmender Anzahl an Grundwasserwärmepumpenanlagen steigt auch das Potenzial an Nutzungsüberlagerungen. Im Rahmen des wasserrechtlichen Genehmigungsverfahrens ist es daher erforderlich, u. a. die von einer Anlage ausgehenden Temperaturfahnen im Grundwasser zu berechnen. In Baden-Württemberg wurde als Erweiterung des für Anlagen $\leq 45.000 \mathrm{kWh} /$ a gültigen Leitfadens des Umweltministeriums Baden-Württemberg das Thermische Online-Modell (TOM) entwickelt. Hierbei handelt es sich um ein vereinfachtes, browserbasiertes, numerisches Grundwassermodell. Ziel dieser Studie ist, die mit TOM berechneten Ausdehnungen von Temperaturfahnen durch mit analytisch und einem kalibrierten numerischen Modell berechnete Temperaturfahnen zu evaluieren. Der Vergleich mit den analytischen Berechnungsmethoden zeigt, dass diese die Ausbreitung der Temperaturfahnen in Relation zu den numerischen Modellen grundsätzlich überschätzen. Für kleine und mittlere Anlagen $(<101 / \mathrm{s})$ wurden mit TOM vergleichbare Ergebnisse wie mit einem kalibrierten numerischen Modell berechnet. Für größere Anlagen ( $>101 / \mathrm{s})$ ist aufgrund der weiträumigeren Ausbreitung der Temperaturfelder die Simulation mit einem kalibrierten numerischen Planungsmodell zu empfehlen.

Schlüsselwörter Geothermie · Grundwasserwärmepumpen · Wärmetransportmodellierung · Konzeptmodell

\section{Calculation of temperature plumes in groundwater with analytical and numerical models}

\begin{abstract}
With increasing numbers of groundwater heat pump systems, the potential for user conflicts is also increasing. As part of the approval process, it is therefore necessary to delineate the temperature plumes in groundwater emanating from those wells. Hence, in the state of Baden-Württemberg, the thermal online model (TOM) was developed as an extension to the existing guidelines for open systems $\leq 45,000 \mathrm{kWh} / \mathrm{a}$. TOM is a simplified, browser-based, numerical groundwater model. The objective of this study is to evaluate the temperature fields calculated using TOM with analytically calculated temperature fields and calculations using a calibrated numerical model. The results show that analytical calculation methods generally overestimate the extent of the temperature plumes in relation to the numerical models. For smaller and medium-sized systems $(<101 / \mathrm{s})$, comparable results were calculated with TOM as with a calibrated numerical model. For larger systems $(>101 / \mathrm{s})$, simulation with a calibrated numerical model is recommended.
\end{abstract}

Keywords Geothermal energy · Groundwater heat pump · Heat transfer modeling · Concept model

Marc Ohmer

marc.ohmer@kit.edu

1 Institut für Angewandte Geowissenschaften (AGW), Karlsruher Institut für Technologie (KIT), Adenauerring 20b, 76131 Karlsruhe, Deutschland

2 Ingenieurgesellschaft Prof. Kobus und Partner GmbH, Wilhelm-Haas-Str. 6, 70771 Leinfelden-Echterdingen, Deutschland
3 Institut für Wasser- und Umweltsystemmodellierung, Universität Stuttgart, Pfaffenwaldring 61, 70569 Stuttgart, Deutschland

4 Fachbereich Wasser und Böden, Landratsamt Breisgau-Hochschwarzwald, Stadtstraße 2, 79104 Freiburg, Deutschland

5 Landesamt für Geologie, Rohstoffe und Bergbau (LGRB), Regierungspräsidium Freiburg, Albertstr. 5, 79104 Freiburg, Deutschland 


\section{Einleitung}

Die Bedeutung von Grundwasserwärmepumpenanlagen (GWWP) zur klimafreundlichen Beheizung und passiven Kühlung von Gebäuden, technischen Anlagen oder Infrastruktureinrichtungen wird in Zukunft weiter zunehmen (VDE 2015). Dabei wird oberflächennahes Grundwasser (GW) aus einem Entnahmebrunnen entnommen, über einen Wärmetauscher Energie entzogen (für Heizzwecke) bzw. zugeführt (für Kühlzwecke) und anschließend über einen Infiltrationsbrunnen wieder zurück in den Grundwasserleiter gegeben (z. B. Stauffer et al. 2014). Die lokale Veränderung der natürlichen Grundwassertemperaturen stellt grundsätzlich einen Eingriff in die natürliche Grundwasserbeschaffenheit dar (Blum et al. 2021). Weiterhin kann es zu Überlagerungen mit anderen Grundwassernutzungen kommen. Dies begründet die Notwendigkeit entsprechender Regelungen, um den Grundwasserschutz zu gewährleisten und Konflikte mit anderen Grundwassernutzungen zu vermeiden.

Einen umfassenden Überblick über die nationale und internationale Rechtslage zur thermischen Nutzung oberflächennaher Geothermie ist in Haehnlein et al. (2010a, 2013) sowie in Tsagarakis et al. (2020) zu finden. Entwicklungskonzepte für das Management von thermischen Ressourcen in städtischen Gebieten wurde u.a. für die Metropolregionen München (Boettcher et al. 2019), Basel (z.B. Epting et al. 2017, 2019; Mueller et al. 2018; Becker und Epting 2021), London (Fry 2009), Turin (Bucci et al. 2017), Zaragoza (García-Gil et al. 2015) sowie in theoretischen Studien (z.B. Pophillat et al. 2018; Piga et al. 2017) und Karlsruhe (Tissen et al. 2019) untersucht. Hierzu wurden unterschiedliche analytische und numerische Modelle verwendet (z.B. Hecht-Méndez et al. 2010). In der Schweiz und u. a. auch in Deutschland wird beispielsweise das vereinfachte numerische Modell „Groundwater Energy Designer“ (GED) zur Planung und Dimensionierung von Grundwasserwärmepumpenanlagen eingesetzt (Poppei und Schwarz 2011).

Das Land Baden-Württemberg hat unter Federführung des Umweltministeriums einen Leitfaden zur Nutzung von Erdwärme mit Grundwasserwärmepumpen herausgegeben (UM BW 2009a). Der Anwendungsbereich beschränkt sich dabei auf kleinere Anlagen mit einem Energieentzug bis $45.000 \mathrm{kWh}$ pro Jahr. Hauptanwendungsbereich dieser Anlagen ist derzeit das Heizen sowie die Warmwasserbereitstellung privater Wohngebäude. Im Leitfaden werden genehmigungsrechtliche Aspekte von GWWP unter Berücksichtigung des Grundwasserschutzes beschrieben und das Genehmigungsverfahren durch die unteren Verwaltungsbehörden schematisch dargestellt. In einer zugehörigen Arbeitshilfe zum Leitfaden (UM BW 2009b) wird die Problematik zur Ermittlung und Berechnung der resultierenden Temperaturfelder behandelt. Hauptausschlusskrite- rien für eine Genehmigung von GWWP sind unverträgliche Veränderungen der natürlichen Grundwassertemperaturen und Konflikte mit anderen Nutzungen. Die Berechnung der Temperaturfelder erfolgt in der Arbeitshilfe mit einer vereinfachten analytischen Näherungslösung für den Jahresund den Winterbetrieb, die auf Anlagen mit einem Energieentzug $<45.000 \mathrm{kWh} / \mathrm{a}$ und Entnahmebreiten der Brunnen unter $30 \mathrm{~m}$ abzielt. Vorgänge wie Interaktionen zwischen Anlagen und Vorflutern, Veränderung der Fließgeschwindigkeit und -strömungsrichtung, konvektive Wärmezu- oder -abflüsse sowie instationärer Anlagenbetrieb werden mit analytischen Lösungen allerdings nicht abgebildet.

Größere Anlagen, wie beispielsweise industrielle Anlagen $(>45.000 \mathrm{kWh} / \mathrm{a})$, können mit dieser Arbeitshilfe nicht behandelt werden und benötigen komplexere Arbeitsansätze zur Wärmetransportmodellierung. Der Einfluss solcher großen Anlagen auf den Temperaturhaushalt des Grundwassers ist vergleichsweise deutlich ausgeprägter und großräumiger. Die daraus resultierenden erweiterten Anforderungen an die Berechnungsmethoden und Modelle umfassen einerseits die Erweiterung der im Modell berücksichtigten physikalischen Prozesse wie z.B. die Auswirkung des instationären Brunnenbetriebs auf Strömung und Transport sowie den Wärmeaustausch mit den liegenden Schichten und der Atmosphäre sowie Mischungsprozesse durch hydrodynamische Dispersion. Andererseits kann es erforderlich sein, Modellparameter, wie z.B. den Durchlässigkeitswert $\left(\mathrm{k}_{\mathrm{f}}\right.$-Wert), räumlich zu variieren und das Strömungsmodell zu kalibrieren. Eine optimale Berechnung von Temperaturfeldern kann letztendlich nur mit dem Einsatz von dreidimensionalen hydraulisch-thermischen Grundwassermodellen mit einer stationären und/oder instationären Kalibrierung der Strömung und des thermischen Modells erreicht werden.

In der technischen Regel DVGW W 107 (A) (2016) werden verschiedene Kategorien von numerischen Grundwassermodellen hinsichtlich der Güte der Abbildung des natürlichen Grundwassersystems, auch Wiedergabetreue genannt, unterschieden. Die niedrigste Wiedergabetreue hat hierbei die Kategorie „Prinzipmodell“. Bei einem Prinzipmodell müssen Strömungs- und Transportprozesse richtig abgebildet sein, es bedarf aber keiner Kalibrierung. Die nächst-höhere Wiedergabetreue hat die Kategorie „Planungsmodell“. Hier ist eine Kalibrierung bzw. ein Modelltest erforderlich. Die höchste Wiedergabetreue erreicht ein „Bewirtschaftungsmodell“, welches aus einem Planungsmodell entsteht, das durch kontinuierliche Datenerhebung und Modellanpassung fortgeschrieben wird. Der Aufwand für Datenerhebung und Modellerstellung sowie die Ansprüche hinsichtlich der Belastbarkeit der Ergebnisse steigen deutlich zwischen diesen Kategorien. In Tab. 1 findet sich eine von uns vorgeschlagene Übersicht des anzuwendenden Berechnungsmodells zur Auswirkung von GWWP in 
Tab. 1 Übersicht der Anforderungen an die unterschiedlichen Berechnungsmodelle zur Auswirkung von GWWP in Abhängigkeit der Anlagengrößen

Table 1 Overview of the requirements for the different calculation models for the impact of GW heat pumps depending on the plant sizes

\begin{tabular}{llll}
\hline Anlagengröße & Klein & Mittel & Groß \\
\hline Entzugsarbeit/Förderrate & $<45.000 \mathrm{kWh} / \mathrm{a}$ & $>45.000 \mathrm{kWh} / \mathrm{a}$ & $>101 / \mathrm{s}$ \\
Berechnungsmodell & $\begin{array}{l}\text { Analytisches Modell } \\
\text { (z. B. LAHM, PAHM) }\end{array}$ & $\begin{array}{l}\text { Prinzipmodell } \\
\text { (z. B. TOM) }\end{array}$ \\
Anforderungen: & & & $\times$ \\
Darstellung des hydrogeologischen Konzeptmodells (HKM) & $\times$ & $\times$ & $\times$ \\
Plausibilitätskontrolle der Wasser- und Energiebilanzen & - & $\times$ & $\times$ \\
Darstellung der gemessenen und simulierten Grundwassergleichen & - & $\times$ & $\times$ \\
Visualisierung der Ergebnisse des thermischen Modells & - & $\times$ & $\times$ \\
Stationäre Kalibrierung des hydraulischen Modells & - & - & $\times$ \\
Instationäre Kalibrierung des hydraulischen Modells & - & - & $\times$ \\
Kalibrierung des thermischen Modells & - & - & $\times$ \\
\hline
\end{tabular}

Abhängigkeit der Anlagengrößen. Die nachfolgend betrachteten Berechnungsmethoden und Modellierungen werden in Anlehnung an diese Kategorien eingeordnet.

Zur Schaffung geeigneter praxisnah und einfach anzuwendender Berechnungsmethoden für Temperaturfelder im Rahmen von Genehmigungsverfahren für große Grundwasserwärmepumpenanlagen mit Jahresentnahmen $>45.000 \mathrm{kWh}$ und Förderraten unter 101/s wurde im Auftrag des Umweltministeriums Baden-Württemberg von der Ingenieurgesellschaft Prof. Kobus und Partner GmbH und dem Lehrstuhl für Hydromechanik und Hydrosystemmodellierung der Universität Stuttgart das webbasierte Thermische Online-Modell (TOM) entwickelt (Kobus und Partner 2020). Hierbei handelt es sich um ein stark vereinfachtes numerisches Grundwassermodell, das online über einen Web-Browser erstellt und ausgewertet werden kann. Als Grundlage verwendet TOM den numerischen (Open-Source) Simulator DuMux (Flemisch et al. 2011; Koch et al. 2020), der im konkreten Fall dieser Anwendung dreidimensionalen Strömungs- und Wärmetransport in porösen Medien berechnet. Dadurch entfallen die oben genannten Einschränkungen analytischer Modelle und die dreidimensionalen Strömungs- und Transportprozesse können abgebildet werden. Darüber hinaus kann ein instationärer Anlagenbetrieb berücksichtigt werden. Weiterhin soll TOM die Simulation von mehreren sich gegenseitig beeinflussenden GWWP-Anlagen ermöglichen. Die Wahl der Eingangsparameter erfolgt dabei bewusst vereinfacht, um auch Anwendern ohne einschlägige Modelliererfahrung die Bedienung zu ermöglichen (Kobus und Partner 2020). Die mit TOM erreichte Wiedergabetreue entspricht ungefähr der Wiedergabetreue eines Prinzipmodells nach DVGW W 107. Somit soll TOM einen Mittelweg zwischen vereinfachten analytischen Modellen und daten- und arbeitsintensiven Planungsmodellen für Anlagen mit einer Entzugsleistung von $>45.000 \mathrm{kWh}$ und Förderraten $<101 / \mathrm{s}$ bieten.
Das Ziel der hier vorgelegten Studie ist, die Ergebnisse des thermischen Online-Modells (TOM) für ein realistisches Fallbeispiel durch Vergleiche mit zwei analytischen Modellen (linear advective heat transport model, LAHM, und planar advective heat transport model, PAHM) sowie einem kalibrierten numerischen Modell (FEFLOW Version 7.4) zu evaluieren. Durch die Berechnung der Strömungs- und Wärmetransportverhältnisse eines Fallbeispiels mit verschiedenen Berechnungsmodellen unterschiedlicher Modellkomplexität, soll aufgezeigt werden, welchen Einfluss die gewählte Methode unter den Anforderungen eines Wasserrechtsantrags auf die berechneten Temperaurfelder hat.

Hierfür wurde ein Untersuchungsgebiet im südlichen Oberrheingraben mit mehreren industriell genutzten Anlagen mit stationären und instationären Anlagenbetrieben zu Kühl- und Heizzwecken und potenziellen Nutzungsüberlagerungen der Anlagen ausgewählt.

\section{Material und Methoden}

\section{Untersuchungsgebiet}

Das Untersuchungsgebiet befindet sich im südlichen Oberrheingraben bei Freiburg. Den geologischen Rahmen bildet die Freiburger Bucht. Diese liegt zwischen dem Kaiserstuhl im Westen und dem Schwarzwald im Osten. Die Freiburger Bucht ist vorwiegend aus quartären Sanden und Kiesen mit Mächtigkeiten von bis zu $240 \mathrm{~m}$ aufgebaut, die sich von den Austrittstälern des Schwarzwalds halbkreisförmig in die Vorbergzone des südlichen Oberrheingrabens vorbauen (LUBW 2006). Die folgende Lockergesteinsabfolge, vom Hangenden zum Liegenden, wird für die stratigraphische Gliederung in LGRB (2007) verwendet:

Die rötlichgrauen, sehr hoch bis hoch durchlässigen Kiese der Neuenburg-Formation (qN) mit wechselnden Sand- 


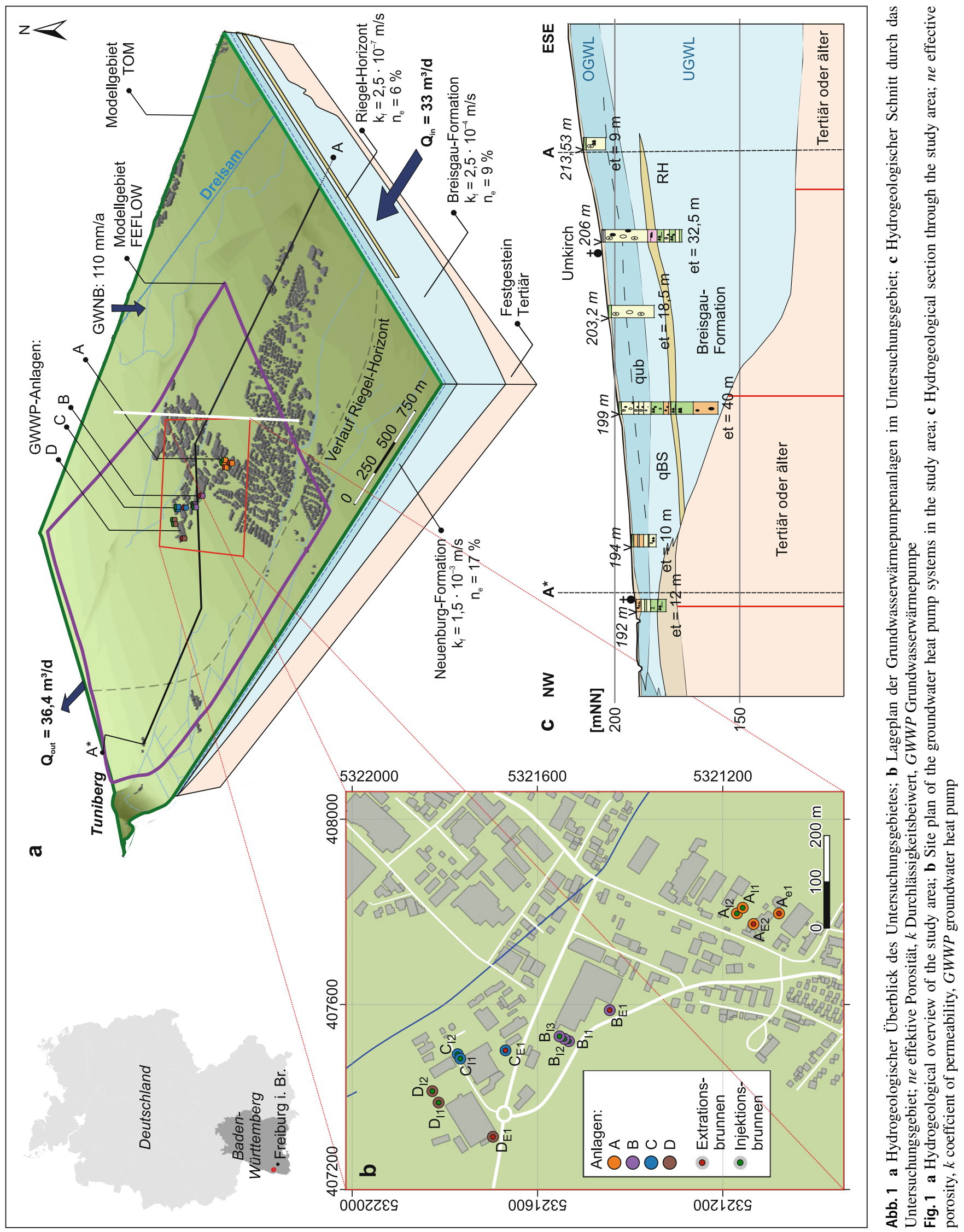


Tab. 2 Schichtspezifische hydrogeologische Parameter aller verwendeter Modelle

Table 2 Layer-specific hydrogeological parameters of all models used

\begin{tabular}{|c|c|c|c|c|c|}
\hline \multirow[t]{2}{*}{ Parameter } & \multirow[t]{2}{*}{ Symbol } & \multirow[t]{2}{*}{ Einheit } & \multicolumn{3}{|l|}{ Werte } \\
\hline & & & LAHM, PAHM & TOM & FEFLOW \\
\hline Initiale Grundwassertemperatur (GWT) & - & {$\left[{ }^{\circ} \mathrm{C}\right]$} & 12,7 & 12,7 & 12,7 \\
\hline Modellschichten & - & - & 1 & 2 & 3 \\
\hline Hydrogeologische Verhältnisse & - & - & gespannt & frei, ungesättig & \\
\hline Grundwasserneubildung & - & {$[\mathrm{mm} / \mathrm{a}]$} & - & 110 & 110 \\
\hline Mittlerer Flurabstand & - & {$[\mathrm{m}]$} & - & 2 & 1,45 \\
\hline \multirow[t]{3}{*}{ Mächtigkeit ${ }^{\mathrm{a}}$} & $M_{N F}$ & {$[\mathrm{~m}]$} & 25,0 & 15,0 & $13,2-8,2^{*}$ \\
\hline & $M_{B F}$ & & & 40,0 & $20,3-53,7 *$ \\
\hline & $M_{R H}$ & & - & - & 2,3 \\
\hline \multirow[t]{3}{*}{ Horizontaler Durchlässigkeitsbeiwert ${ }^{\mathrm{b}}$} & $k_{f x y, N F}$ & {$[\mathrm{~m} / \mathrm{s}]$} & $1,0 \cdot 10^{-3}$ & $1,5 \cdot 10^{-3}$ & $1-50 \cdot 10^{-4 *}$ \\
\hline & $k_{f x y, B F}$ & & & $2,5 \cdot 10^{-4}$ & $2,5 \cdot 10^{-4}$ \\
\hline & $k_{f x y, R H}$ & & - & - & $1,0 \cdot 10^{-7}$ \\
\hline \multirow[t]{3}{*}{ Vertikaler Durchlässigkeitsbeiwert ${ }^{c}$} & $k_{f z, N F}$ & {$[\mathrm{~m} / \mathrm{s}]$} & - & - & $0,2-10 \cdot 10^{-4}$ \\
\hline & $k_{f z, B F}$ & & & & $0,5 \cdot 10^{-4}$ \\
\hline & $k_{f z, R H}$ & & & & $1,0 \cdot 10^{-7}$ \\
\hline \multirow[t]{3}{*}{ Effektive Porosität ${ }^{\mathrm{d}}$} & $n_{e, N F}$ & {$[-]$} & 0,14 & 0,17 & 0,17 \\
\hline & $n_{e, B F}$ & & & 0,09 & 0,09 \\
\hline & $n_{e, R H}$ & & - & - & 0,05 \\
\hline \multirow[t]{3}{*}{ Wärmeleitfähigkeit des porösen Mediums ${ }^{\mathrm{e}}$} & $\lambda_{N F}$ & {$[\mathrm{~W} / \mathrm{K} \cdot \mathrm{m}]$} & 2,28 & 2,40 & 2,40 \\
\hline & $\lambda_{B F}$ & & & 2,10 & 2,10 \\
\hline & $\lambda_{R H}$ & & - & - & 1,80 \\
\hline \multirow{3}{*}{$\begin{array}{l}\text { Volumenbezogene spezifische Wärmekapazität } \\
\text { des porösen Mediums }{ }^{\mathrm{f}}\end{array}$} & $c_{N F}$ & {$\left[\mathrm{MJ} / \mathrm{K} \cdot \mathrm{m}^{3}\right]$} & 2,93 & 2,90 & 2,90 \\
\hline & $c_{B F}$ & & & 3,00 & 3,00 \\
\hline & $c_{R H}$ & & - & - & 3,40 \\
\hline Wärmeleitfähigkeit des Grundwassers & $\lambda_{w}$ & {$[\mathrm{~W} / \mathrm{K} \cdot \mathrm{m}]$} & - & 0,6 & 0,6 \\
\hline $\begin{array}{l}\text { Volumenbezogene spezifische Wärmekapazität } \\
\text { des Grundwassers }\end{array}$ & $c_{W}$ & {$\left[\mathrm{MJ} / \mathrm{K} \cdot \mathrm{m}^{3}\right]$} & 4,17 & 4,17 & 4,17 \\
\hline Longitudinale Dispersion & $\alpha_{L}$ & {$[\mathrm{~m}]$} & 5,0 & 5,0 & 5,0 \\
\hline Transversale Dispersion & $\alpha_{T}$ & & 0,5 & 0,5 & 0,5 \\
\hline \multirow[t]{3}{*}{ Retardationsfaktor } & $R_{N F}$ & {$[-]$} & 4,87 & 4,09 & 4,09 \\
\hline & $R_{B F}$ & & & 4,2 & 4,2 \\
\hline & $R_{R H}$ & & - & - & 13,78 \\
\hline Gitter-Péclet-Zahl & $P e$ & {$[-]$} & - & 1 & $<0,05$ \\
\hline
\end{tabular}

$N F$ Neuenburg-Formation, $B F$ Breisgau-Formation, $R H$ Riegel-Horizont

* Wertebereich nach LGRB (2007)

${ }^{\mathrm{a}} \mathrm{M}_{\mathrm{NF}}, \mathrm{M}_{\mathrm{BF}}, \mathrm{M}_{\mathrm{RH}}$ : LGRB (2007)

${ }^{\mathrm{b}} \mathrm{k}_{\mathrm{fxy}}$, NF: LGRB (2007), kfxy, BF: Frey (2016), kfxy, RH: DIN e. V. (1998)

${ }^{c}$ nach Umweltministerium BW (2004)

${ }^{d} n_{e}, N F, n_{e}, B F:$ nach Marotz (1968), $n_{e}, R_{H}$ : nach Garling und Dittrich (1979)

e Dalla Santa et al. (2017), Márquez et al. (2016), VDI 4640 (2010)

${ }^{\mathrm{f}}$ Márquez et al. (2016), VDI 4640 (2010), Abu-Hamdeh (2003), Stathers und Spittlehouse, (1990)

gehalten bilden den ungespannten Oberen Porengrundwasserleiter (OGWL). Die Neuenburg-Formation besitzt stellenweise Einschaltungen von Hochflutsedimenten mit geringerer hydraulischer Durchlässigkeit. Im Gebiet des Fallbeispiels hat die Neuenburg-Formation Mächtigkeiten zwischen 13 und $18 \mathrm{~m}$ und hydraulische Durchlässigkeiten zwischen 0,1 und $2 \cdot 10^{-3} \mathrm{~m} / \mathrm{s}$. Darunter folgt die Breisgau-Formation (qBS). Hierbei handelt es sich um meist stark bis sehr stark verwitterte, unterschiedlich dicht gelagerte, san- dig-schluffige Kiese, herabtransportiert aus den östlich gelegen Höhen des Schwarzwalds, die den Unteren Grundwasserleiter (UGWL) in der Freiburger Bucht bilden. Stellenweise sind geringmächtige Schlufflinsen eingelagert. Die hydraulische Durchlässigkeit der Breisgau-Formation variiert zwischen 0,07 und $11,2 \cdot 10^{-3} \mathrm{~m} / \mathrm{s}$. Im Bereich des Untersuchungsgebiets liegt der Mittelwert bei $2,5 \cdot 10^{-4} \mathrm{~m} / \mathrm{s}$. Die Mächtigkeit beträgt im Gebiet des Fallbeispiels ca. $100 \mathrm{~m}$. Der feinklastische Riegel-Horizont (qRH) ist eine hydrau- 
lisch wirksame Lage innerhalb des oberen Abschnitts der Breisgau-Formation innerhalb der Freiburger Bucht. Er besteht aus schwach feinsandigen schluffigen Tonen und tonigen Schluffen. Der Horizont hat im Untersuchungsgebiet eine durchschnittliche Mächtigkeit von 2 bis $3 \mathrm{~m}$. Die Feinsedimente des Horizonts besitzen eine hydraulische Durchlässigkeit im Bereich von $10^{-7} \mathrm{~m} / \mathrm{s}$. Der Festgesteinsuntergrund $(\mathrm{t})$ besteht im Untersuchungsgebiet aus gering durchlässigen tertiären Tonmergel- und Mergelsteinen (LGRB 2007). In Abb. 1 und in Tab. 2. finden sich die für diese Studie verwendeten schichtspezifischen hydrogeologischen Parameter.

\section{Modellaufbau und Modellparametrisierung}

Das Hydrogeologische Konzeptmodell (HKM) basiert auf den großräumigen Daten zum hydrogeologischen Bau des Oberrheingrabens (LGRB 2007). Anzahl und Mächtigkeiten der in den Modellen (FEFLOW, TOM, LAHM/PAHM) implementierten Schichten sowie die schichtspezifischen Parameter sind in Tab. 2 aufgeführt.

Für die analytischen Lösungen zur Temperaturfeldberechnung wurden die Parameter der Neuenburg- und Breisgau-Formation gemäß ihrer Mächtigkeit gewichtet gemittelt und in ein 1-Schichtmodell übertragen. Da es sich bei den im nächsten Abschnitt erläuterten Anlagen um unvollkommene Brunnen handelt, wurde gemäß UM BW (2009a) die dreidimensionale Trennstromfläche der Rückgabebrunnen ermittelt und als Ersatztiefe abgeschätzt. Die Abbildung der Anlagen erfolgt bei den analytischen Modellen stationär über den Jahresmittelwert. Die Kühlung der Anlage D wurde gemäß des Leitfadens in beiden analytischen Methoden dabei vernachlässigt.

Die numerischen Wärmeberechnungen von TOM und FEFLOW beruhen beide auf ungesättigten Strömungsmodellen. Da TOM derzeit auf die Berücksichtigung von zwei Modellschichten limitiert ist, wurde der geringmächtige Riegel-Horizont in der Berechnung nicht berücksichtigt. Ein konduktiver Wärmeaustausch mit der Atmosphäre über die ungesättigte Zone ist in TOM als Festpotenzialrand mit konstanter Druckhöhe (RB 1. Art, Dirichlet) implementiert (Mittlere Tagesmitteltemperatur von $12,7^{\circ} \mathrm{C}$ ). Die Abgrenzung des Modellgebietes in FEFLOW erfolgte unter Verwendung des Mittelwasserstand-Grundwassergleichenplans 1986 des Regionalverbands südlicher Oberrhein (RVSO 1986). Die nordwestliche und die südöstliche Modellgrenze wurden als Festpotenzialrand auf Basis der GWIsolinien $206 \mathrm{mNN}$ (Südost) und $190 \mathrm{mNN}$ (Nordwest) implementiert. Sie sind senkrecht zu den Grundwassergleichen konstruiert, sodass kein Grenzaustausch stattfindet (RB 2. Art, Neumann, mit $Q=0$ ). Die Geometrie des FEFLOW-Modells ist keilförmig. Die Gesamtmächtigkeit nimmt von $75 \mathrm{~m}$, am südöstlichen Rand, gegen Nordwes- ten auf $20 \mathrm{~m}$ ab. Die Abschätzung des Wärmeaustausches mit der Atmosphäre erfolgte zum einen über eine Dirichlet-Randbedingung mit einer mittleren Tagestemperatur von $12,7^{\circ} \mathrm{C}$ entlang der ersten Modellslice als auch, zu Vergleichszwecken, über eine Cauchy-Randbedingung mit einer Transferrate $\Phi$ nach Gl. 10, als Quotient aus der mittleren Wärmeleitfähigkeit der ungesättigten Zone $\lambda_{N F}$ von $2,40[\mathrm{~W} / \mathrm{K} \cdot \mathrm{m}]$ und räumlich berechnetem Flurabstand $d$ bei MW 1986 abgeschätzt. Die Verwendung von mittleren Luft- und GW-Jahresdurchschnittstemperaturen bei FEFLOW soll der besseren Vergleichbarkeit mit den mit TOM erzielten Ergebnissen dienen. Je nach saisonaler Nutzung und Temperatur-Variabilität kann dies allerdings einen großen Einfluss auf die Temperaturfahnen haben.

Die Abbildung der Anlagen erfolgt für die numerischen Modelle TOM und FEFLOW instationär mit monatlichen Zeitschritten und Monatsmittelwerten für einen Berechnungszeitraum von 5 Jahren. Die Brunnen sind gemäß ihrer Dimensionierung und Filterstrecke und -tiefe in die Modelle auf Basis ihrer mittleren monatlichen Entnahmeraten eingebaut.

\section{Grundwasserwärmepumpenanlagen}

Als Fallbeispiel dienen vier industriell genutzte Anlagen in Umkirch bei Freiburg im Breisgau. Die Anlagen A und B werden zu reinen Kühlzwecken (industrielle Kühlwasseranlagen) betrieben (Abb. 2). Die Anlagen B und C sind mit stationären Raten ganzjährig in Betrieb, wobei Anlage C ausschließlich zu Heizzwecken eingesetzt wird. Anlage D wird kombiniert zur Kühlung im Sommer und Heizung im Winter eingesetzt.

Alle Brunnen erschließen den Oberen Grundwasserleiter der Neuenburg-Formation. Nur bei der Anlage B reichen die Brunnen und deren Filterstrecken drei Meter in die Breisgau-Formation hinein. Alle Anlagen übersteigen mit ihren jährlichen mittleren Energieentzügen deutlich den Nutzungsumfang von $45.000 \mathrm{kWh}$ pro Jahr und liegen damit außerhalb des Geltungsbereichs des Leitfadens für Baden-Württemberg (UM BW 2009a). Die in Monatsschritten zusammengefassten Entnahmen (Tagesmittelwerte) sind in der Abb. 2 dokumentiert. Die Art der Temperaturnutzung des Grundwassers ist in der Abb. 2 jeweils auf der rechten Ordinate abgebildet (positive Werte: Kühlung, negative Werte: Heizung). Die Entnahmeraten entsprechen den Rückgaberaten. Die angegebenen Temperaturdifferenzen gelten für die Rückgabe in den Grundwasserleiter. Die in der Abb. 2 gemachten Angaben gelten summarisch für die jeweilige Gesamtanlage. Den größten Energieumsatz hat, mit $2123 \mathrm{GW}$ pro Jahr, Anlage B. 


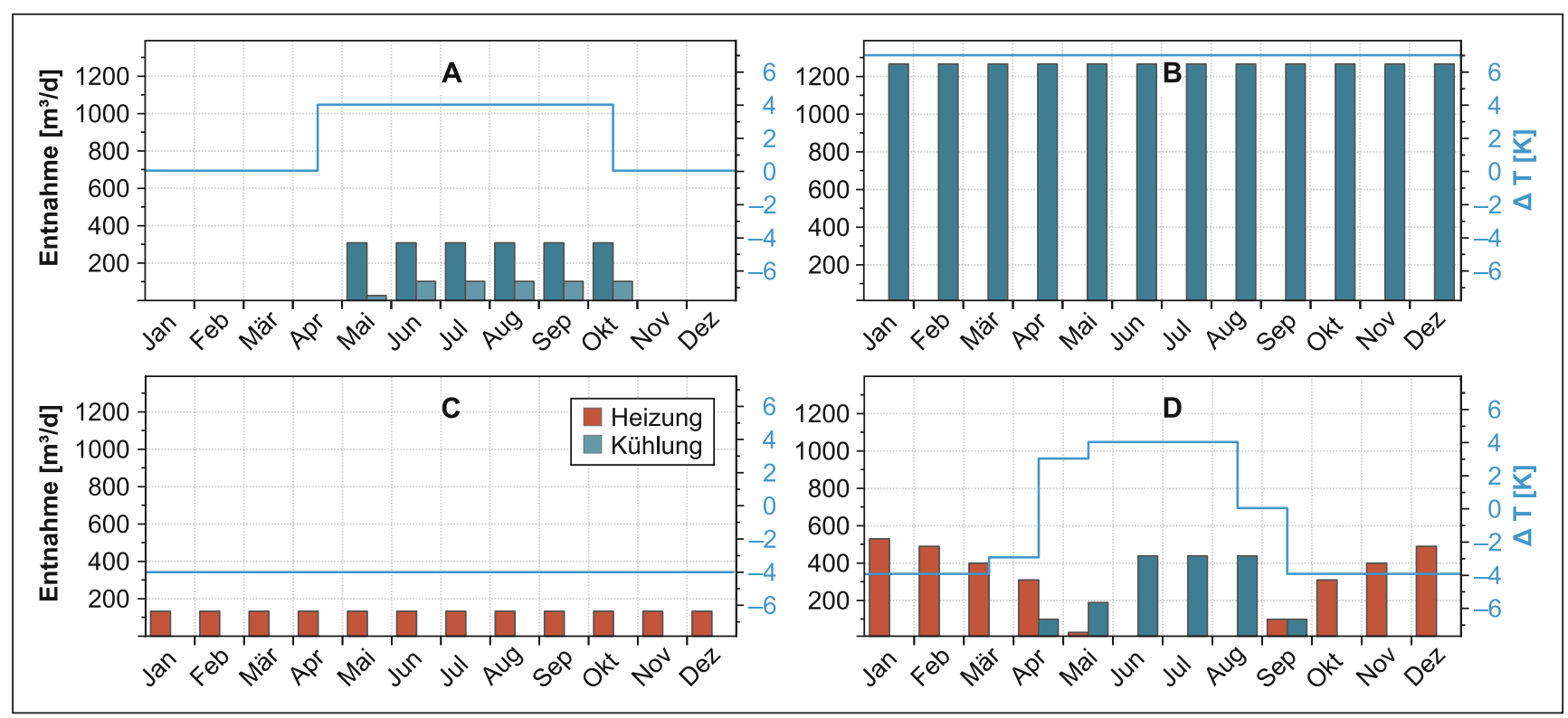

Abb. 2 Monatliche Entnahmeraten der Grundwasserwärmepumpenanlagen im Untersuchungsgebiet

Fig. 2 Monthly extraction rates of the groundwater heat pump systems in the study area

\section{Grundlagen der Wärmeausbreitung}

Der Wärmetransport im Grundwasser erfolgt im Wesentlichen durch Wärmeleitung (Wärmediffusion/Konduktion), Advektion/Konvektion und Dispersion (Sauty 1980). Grundlagen des Stoff- und Wärmetransports finden sich z.B. in Bear (1972), De Marsily (1980), Häfner et al. (1992) und Stauffer et al. (2014). Bei der Berechnung wird grundsätzlich zwischen dem konduktiven und dem konvektiven Wärmetransport sowie zwischen stationären und instationären Bedingungen unterschieden (Hähnlein et al. 2010b).

\section{Analytische Modelle}

Analytische Modelle beruhen auf analytischen Lösungen des Anfangs- und Randwertproblems, welches sich aus den berücksichtigten physikalischen Prozessen und den daraus resultierenden Differenzialgleichungen ergibt (Kinzelbach 1987). Sie bieten dann die Möglichkeit, mit wenigen Eingabeparametern und geringem Berechnungsaufwand die hydraulischen und thermischen Prozesse im idealisierten und isotropen Grundwasserleiter abzuschätzen. Vorgänge wie Interaktionen zwischen Anlagen und Vorflutern, Veränderung der Fließgeschwindigkeit und -strömungsrichtung und konvektive Wärmezu- oder -abflüsse sowie instationärer Anlagenbetrieb können dabei jedoch nicht berücksichtigt werden. Im vorliegenden Fall wurden die analytischen Berechnungen mit den Verfahren LAHM und PAHM durchgeführt (s. unten). Die Berechnungen erfolgen bei LAHM und PAHM entsprechend des im Leitfaden (UM BW 2009b) ge- nannten Lastfalls „Jahresmittelwert“. Dieser soll die langfristige Beeinflussung, bei Dauerbetrieb einer Anlage unter Annahme einer konstanten Entnahmerate und Spreizung, beschreiben.

\section{Linear advective heat transport model (LAHM)}

Das als LAHM bezeichnete analytische Berechnungsverfahren wurde von Kinzelbach (1987) entwickelt und wird in der Arbeitshilfe zum ,Leitfaden zur Nutzung von Erdwärme mit Grundwasserwärmepumpen für Ein- und Zweifamilienhäuser oder Anlagen mit Energieentzug bis zirka 45.000 kWh pro Jahr“" verwendet (UM BW 2009b). Gemäß dem Leitfaden ist die Anwendung des LAHM auf Fließgeschwindigkeiten von über $1 \mathrm{~m} / \mathrm{Tag}$ (für den stationären Fall) und für Anlagen mit Energieentzug unter $45.000 \mathrm{kWh}$ pro Jahr begrenzt. Beschrieben wird das Temperaturfeld einer kontinuierlichen Punktquelle infolge einer konvektiven und dispersiven Ausbreitung in einem homogenen, gespannten Porengrundwasserleiter bei instationären Bedingungen:

$$
\begin{aligned}
\Delta T(x, y, t)= & \frac{Q \cdot \Delta T_{\mathrm{inj}}}{4 \cdot n_{e} \cdot M \cdot v_{a} \cdot \sqrt{\pi \cdot \alpha_{T}}} \\
& \cdot \exp \left(\frac{x-r}{2 \cdot \alpha_{L}}\right) \cdot \frac{1}{\sqrt{r}} \\
& \cdot \operatorname{erfc}\left(\frac{r-v_{a} \cdot t / R}{2 \cdot \sqrt{v_{a} \cdot \alpha_{L} \cdot t / R}}\right)
\end{aligned}
$$


mit:

$r=\sqrt{x^{2}+y^{2} \frac{\alpha_{L}}{\alpha_{T}}}$

$\Delta T:$ Gesuchte Isotherme als Differenz zur unbeeinflussten Grundwassertemperatur [K]

$Q: \quad$ Injektionsrate $\left[\mathrm{m}^{3} / \mathrm{s}\right]$

$T_{i n j}$ : Differenz zwischen der Injektionstemperatur und der unbeeinflussten Grundwassertemperatur [K]

$n_{e}: \quad$ effektive Porosität [-]

$M: \quad$ genutzte, grundwassererfüllte Mächtigkeit [m]

$v_{a}$ : Abstandsgeschwindigkeit [m/s]

$\alpha_{L, T}$ : Längs- und Querdispersivität [m]

$x, y$ : Längs- und Querkoordinaten [m]

$t: \quad$ Zeit [s]

$R: \quad$ Retardation [-]

$r: \quad$ radialer Abstand vom Injektionsbrunnen [m]

\section{Planar advective heat transport model (PAHM)}

PAHM wurde von Domenico und Robbins (1985) als ein zweidimensionales Transportmodell für instationäre Bedingungen mit einer homogenen, parallel gerichteten Grundwasserströmung entwickelt und von Hähnlein et al. (2010b) für die Berechnung zur Ausdehnung der Temperaturfahne umformuliert. Die Anwendung ist begrenzt auf Grundwasserfließgeschwindigkeiten zwischen 1 und $10 \mathrm{~m}$ pro Tag. Eine umfassende Erläuterung findet sich in Pophillat et al. (2018). Im Gegensatz zu LAHM wird der Wärmeeintrag nicht als Punktquelle, sondern als vertikale Fläche senkrecht zur Grundwasserströmung in einem homogenen, gespannten Grundwasserleiter abgebildet. Dadurch sollen große lokale hydraulische Gradienten im Bereich des Infiltrationsbrunnens besser repräsentiert werden (Pophillat et al. 2018).

$$
\begin{aligned}
& \Delta T(x, y, t)=\left(\frac{\Delta T_{0}}{4}\right) \cdot \operatorname{erfc}\left(\frac{R \cdot x-v_{a} \cdot t}{2 \cdot \sqrt{D_{L} \cdot R \cdot t}}\right) \\
& \cdot\left\{\operatorname{erf}\left[\frac{y+\frac{Y}{2}}{2 \cdot \sqrt{D_{T} \cdot \frac{x}{v_{a}}}}\right]-\operatorname{erf}\left[\frac{y-\frac{Y}{2}}{2 \cdot \sqrt{D_{T} \cdot \frac{x}{v_{a}}}}\right]\right\}
\end{aligned}
$$

$\Delta T_{0}=\frac{F_{0}}{v_{a} \cdot n_{e} \cdot c_{w} \cdot Y}$

$F_{0}=\frac{q h}{M}$

$q_{h}=\Delta T_{\text {inj }} \cdot c_{w} \cdot Q$

$D_{L, T}=\frac{\lambda_{m}}{n_{e} \cdot c_{w}}+\alpha_{L, T} \cdot v_{a}$

$Y_{\max }=\frac{Q}{M \cdot v_{a} \cdot n_{e}}$ mit:

$\Delta T:$ Gesuchte Isotherme als Differenz zur unbeeinflussten Grundwassertemperatur [K]

$\Delta T_{0}$ : Temperaturdifferenz am Injektionspunkt [K]

$\Delta T_{i n j}$ :Differenz Injektionstemperatur und unbeeinflusste Grundwassertemperatur [K]

$F_{0}$ : Energieeinspeisung über die Länge des Brunnens [W/m]

$q_{h}: \quad$ injizierte Wärmeenergie [W]

$c_{w}$ : Volumenbezogene spezifische Wärmekapazität von Wasser $\left[\mathrm{J} / \mathrm{K} \cdot \mathrm{m}^{3}\right]$

$Q: \quad$ Injektionsrate $\left[\mathrm{m}^{3} / \mathrm{s}\right]$

$M$ : Aquifermächtigkeit [m]

$v_{a}$ : Abstandsgeschwindigkeit $[\mathrm{m} / \mathrm{s}]$

$n_{e}: \quad$ effektive Porosität [-]

$Y$ : Länge der Quelle [m]

$x, y$ : Längs- und Querkoordinaten [m]

$t: \quad$ Zeit [s]

$R: \quad$ Retardation [-]

$D_{L, T}$ : longitudinaler und transversaler hydrodynamischer Dispersionskoeffizient $\left[\mathrm{m}^{2} / \mathrm{s}\right]$

$\alpha_{L, T}$ : Längs- und Querdispersivität [m]

$\lambda_{m}$ : Wärmeleitfähigkeit des porösen Mediums $[\mathrm{W} / \mathrm{m} \cdot \mathrm{K}]$

\section{Numerische Modelle}

\section{Software FEFLOW}

FEFLOW ist ein weit verbreitetes, vielseitiges Finite-Elemente-Simulationssystem zur zwei- und dreidimensionalen Berechnung von Strömungs-, Wärme- und Stofftransportprozessen im Grundwasser. Die entsprechenden Gleichungen für die Wärmetransportmodellierung sind in Diersch (2014) beschrieben. FEFLOW wurde bereits in einer Vielzahl von verwandten Studien zur Simulation der thermischen Grundwassernutzung durch GWWP und durch Erdwärmesonden angewendet (z.B. Wagner et al. 2012; Lo Russo et al. 2014; Rivera et al. 2016; Steiner et al. 2016; Attard et al. 2016; Epting et al. 2017; Pophillat et al. 2018, 2020, Becker und Epting 2021). FEFLOW ermöglicht die Berechnung von gespannten und ungespannten Verhältnissen in gesättigten, variabel gesättigten und ungesättigten porösen Medien. Die Berechnung in dieser Studie erfolgte über die Richards-Gleichung für ungesättigte Strömungen. Die verwendeten Modellgeometrien und die Parametrisierung finden sich in Tab. 3. Die Berechnung der effektiven Wärmeleitfähigkeit erfolgt in FEFLOW als arithmetisches Mittel der beteiligten Medien nach der Gl. 9 (Diersch 2014):

$\lambda_{\mathrm{eff}}=n_{e} \cdot \lambda_{w}+\left(1-n_{e}\right) \cdot \lambda_{s}$ 
Tab. 3 Technische Daten der Grundwasserwärmepumpenanlagen im Untersuchungsgebiet

Table 3 Technical data of the groundwater heat pump systems in the study area

\begin{tabular}{|c|c|c|c|c|}
\hline Anlage & $\begin{array}{l}\text { Mittlere Entnahme-/Infiltrationsrate } \\
{[1 / s]}\end{array}$ & $\begin{array}{l}\text { Mittlere Leistung }{ }^{\mathrm{a}} \\
{[\mathrm{kW}]}\end{array}$ & $\begin{array}{l}\text { Betriebsdauer } \\
\text { [Stunden pro Jahr] }\end{array}$ & $\begin{array}{l}\text { Mittlerer Energiebedarf pro Jahr } \\
{[\mathrm{kWh} / \mathrm{a}]}\end{array}$ \\
\hline $\bar{A}$ & 2,3 & 38 & 2555 & 96.256 \\
\hline B & 14,7 & 431 & 4927 & 2.123 .772 \\
\hline $\mathrm{C}$ & 1,5 & 26 & 4320 & 111.767 \\
\hline D & 4,5 & 75 & 4380 & 327.892 \\
\hline
\end{tabular}

${ }^{a}$ nach UM BW (2009b)

mit:

$\lambda_{\text {eff: }} \quad$ effektive Wärmeleitfähigkeit des Aquifers $[\mathrm{W} / \mathrm{K} \cdot \mathrm{m}]$

$\lambda_{s}$ : Wärmeleitfähigkeit poröses Medium $[\mathrm{W} / \mathrm{K} \cdot \mathrm{m}]$

$\lambda_{w}$ : Wärmeleitfähigkeit Wasser $[\mathrm{W} / \mathrm{K} \cdot \mathrm{m}]$

$n_{e}$ : effektive Porosität [-]

FEFLOW erlaubt die Abschätzung des Energieverlustes bzw. -gewinns mit der Atmosphäre entweder als DirichletRandbedingungen als Festpotenzial oder Cauchy-Randbedingung mittels einer Transferrate (3. Art, Cauchy-Randbedingung).

$Q_{\mathrm{Wärme}}=\mathrm{A} \Phi\left(T_{\mathrm{Luft}}-T_{G W}\right)$

Die Transferrate ist der Wärmedurchgangskoeffizient $\Phi$, der die Eigenschaften der ungesättigten Zone zwischen der Lufttemperatur $T_{\text {Luft }}$ und der Grundwassertemperatur $T_{G W}$ beschreibt. Der Wärmedurchgangskoeffizient $\Phi$ für ein Material ist definiert als Quotient der Wärmeleitfähigkeit der ungesättigten Zone $\lambda$ und ihrer Mächtigkeit $d$.

\section{Thermisches Online-Modell (TOM)}

TOM ist eine numerische Simulationsplattform, welche als Webanwendung zur Simulation von Wärmetransportvorgängen von GWWP-Anlagen (offene Systeme) in Grundwasserleitern genutzt werden kann. Sie wurde von Kobus und Partner (2020) im Auftrag des Umweltministeriums Baden-Württemberg entwickelt und ist eine Erweiterung der im Leitfaden des Landes Baden-Württemberg (UM BW 2009a) vorgegebenen Methode zur Berechnung von Temperaturfeldern für Anlagen über $45.000 \mathrm{kWh}$ pro Jahr. Durch TOM sollen die Einschränkungen der analytischen Lösungen weitgehend entfallen. Des Weiteren kann die vertikale Wärmeausbreitung berücksichtigt werden, und es ist möglich, mehrere sich gegenseitig beeinflussende, instationär betriebene Anlagen zu berücksichtigen. TOM verwendet als numerisches Simulationsmodell DuMu${ }^{\mathrm{x}}$, einen Open-Source Simulator für Strömungs- und Stofftransportprozesse in porösen Medien (Flemisch et al. 2011, Koch et al. 2020). Realisierbar ist sowohl die Modellierung von gespannten Verhältnissen (Ein-Phasen-Modell mit Wasser als einziger Fluid-Phase und konstanter Transmissivität bei stationärem Strömungsfeld, konstante Transmissivität unabhängig vom GW-Stand, Berechnung von stationärem Strömungsfeld für jeden Monat) als auch von ungespannten Verhältnissen (ungesättigtes Zwei-PhasenModell, Berechnung mit Richards-Gleichung, Transmissivität abhängig vom Grundwasserstand bei instationärem Strömungsfeld für jeden Monat). Der Wärmetransport berücksichtigt die Prozesse Advektion, hydrodynamische Dispersion, Konduktion und Wärmespeicherung in Gestein und Wasser. Der konduktive Wärmeaustausch mit der Atmosphäre und der unterlagernden Schicht wird ebenfalls berücksichtigt. Monatlich variierende Brunnenraten sind implementierbar. Basierend auf den Eingangsparametern wird automatisch ein Verfeinerungsbereich des Modellnetzes erzeugt, welches vorab automatisch mittels des PAHMModells bestimmt wird. Der konduktive Wärmeaustausch mit der Atmosphäre ist über einen Festpotenzialrand (Dirichlet-Randbedingung) implementiert. Eine Ausgabe der Ergebnisse erfolgt als standardisierter Ergebnisbericht mit den verwendeten Inputparametern und einem Ergebnisteil inklusive Geodatenformat Shapefile.

Die Berechnung in dieser Studie erfolgte über die Richard-Gleichung für ungesättigte Strömungen. Die effektive Wärmeleitfähigkeit des Aquifers wird in TOM als geometrisches Mittel nach Gl. 10 (Kobus und Partner 2020) berechnet. Dies liefert bei gleichen Eingangsparametern geringere effektive Wärmeleitfähigkeiten als das in FEFLOW verwendete Modell. In Menberg et al. (2013) wird mit dem geometrischen Mittel eine bessere Übereinstimmung zwischen berechneten und im Labor gemessenen Wärmeleitfähigkeiten erzielt.

$\lambda_{\text {eff }}=\lambda_{s}^{(1-n)} \cdot \lambda_{w}^{n}$

mit:

$\lambda_{\text {eff: }} \quad$ effektive Wärmeleitfähigkeit $[\mathrm{W} / \mathrm{K} \cdot \mathrm{m}]$

$\lambda_{s}: \quad$ Wärmeleitfähigkeit Gestein $[\mathrm{W} / \mathrm{K} \cdot \mathrm{m}]$

$\lambda_{w}$ : Wärmeleitfähigkeit Wasser $[\mathrm{W} / \mathrm{K} \cdot \mathrm{m}]$

$n_{e}: \quad$ effektive Porosität [-] 


\section{Stationäre Modellkalibrierung}

Als Basis für die beiden numerischen Modelle wurde der Mittelwasserstand-Grundwassergleichenplan 1986 des Regionalverbands südlicher Oberrhein (RVSO 1986) verwendet (Abb. 3). Innerhalb des Modellgebiets befindet sich nur eine Grundwassermessstelle. Daher wurden im Bereich des FEFLOW-Modellraums randomisiert 300 Punkte als digitale Behelfsmessstellen mit extrahierten Werten des interpolierten Grundwassergleichenplans bei der Kalibrierung verwendet. Bei FEFLOW wurde eine Detailkalibrierung über die Anpassung der Durchlässigkeitsbeiwerte innerhalb des von LGRB (2007) genannten Bereichs durchgeführt. Bei TOM wird das Fließfeld vom Bearbeiter definiert, indem ein Grundwasserstand für einen Bezugspunkt, der hydraulische Gradient und die Strömungsrichtung, der Aquiferaufbau (max. 2 Schichten) und die hydraulischen Durchlässigkeiten (konstant für die jeweilige Schicht) eingegeben werden. Das Strömungsfeld wird in TOM mit mittleren monatlichen Brunnenraten zunächst für den stationären Fall berechnet. Der mittlere relative Fehler zwischen den gemessenen und den berechneten Grundwassergleichen (nMAE) beträgt bei TOM 2,3\% und bei FEFLOW 1,3\%. Daher kann bei beiden Modellen von einer guten stationären Modellanpassung gesprochen werden (DVGW W 107).

\section{Ergebnisse und Diskussion}

Abb. 4 zeigt die berechneten Temperaturfelder der zwei analytischen Modelle (LAHM und PAHM), des numerischen Prinzipmodells (TOM) und des numerischen Planungsmodells (FEFLOW) für die Anlagen A-D im Untersuchungsgebiet. In den analytischen Lösungen werden die einzelnen Anlagen isoliert betrachtet, eine mögliche Wechselwirkung mit anderen Anlagen wird dabei vernachlässigt bzw. kann nicht abgebildet werden. Die Beurteilung der Anlagen erfolgt gemäß den Mindestanforderungen des Leitfadens zur Nutzung von GWWP in Baden-Württemberg (UM BW 2009a). Die Temperaturfeldberechnung mit analytischen Methoden erfolgt für den Lastfall mithilfe eines Jahresmittelwertes. Für die Anlagen B und C ergeben sich aufgrund der konstanten Rate keine Unterschiede. Bei der Betrachtung des Winterbetriebs der Anlagen A und D ergeben sich mit Sicherheit größere Fahnen. Die Berechnung der numerischen Lösungen erfolgt bei gleichzeitigem Be-

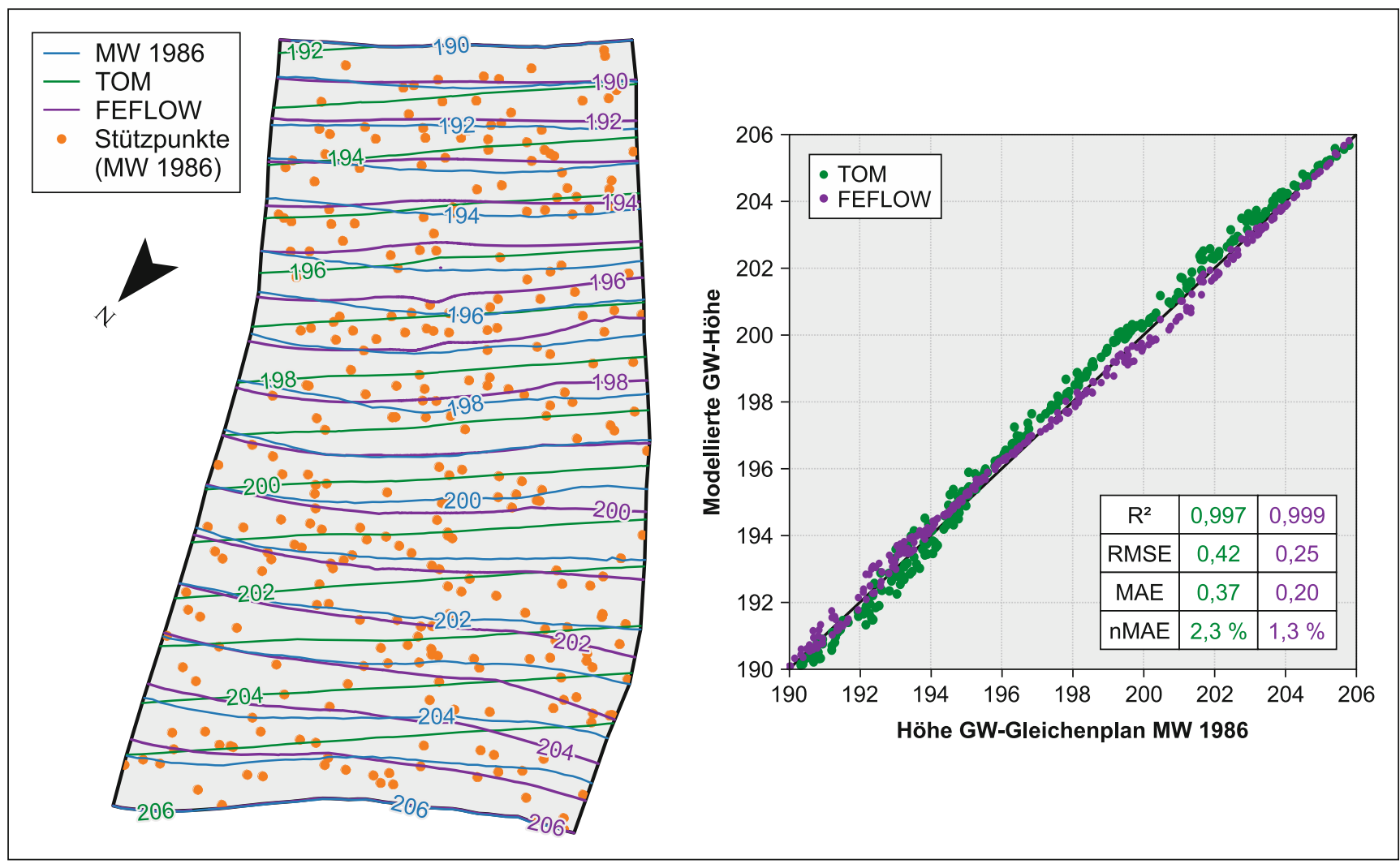

Abb. 3 Vergleich der Grundwasserhöhen, basierend auf einem Grundwassergleichenplan des mittleren Grundwasserstands aus dem Jahr 1986 (blau), des TOM-Modells (grün) und des kalibrierten FEFLOW-Modells (lila)

Fig. 3 Comparison of groundwater levels based on a groundwater contour map of the mean groundwater level from 1986 (blue), the calibrated FEFLOW model (purple) and the TOM model (green) 
trieb aller Anlagen im Modell. Die mit TOM und FEFLOW berechneten Temperaturfelder zeigen die maximale Abkühlung und maximale Erwärmung über alle Modelltiefen während des Simulationszeitraums von 5 Jahren. Aufgrund der Überlagerung bei den numerischen Ansätzen werden die Fahnen der Anlagen C und D zusammengefasst. Die nach den analytischen Ansätzen berechneten Temperaturfelder wurden in das Strömungsfeld hineinprojiziert.
- Anlage A (Kühlung, Betrieb Mai-Oktober, $\Delta \mathrm{T}$ : $4 \mathrm{~K}$, 96.256kWh/a): Bei keinem der vier Modellergebnisse liegt eine unmittelbare Beeinflussung $(\Delta \mathrm{T}>1 \mathrm{~K})$ von im Abstrom gelegenen Anlagen B-D durch die Wärmefahne der Anlage A vor. Die stationäre Anlagenbetrachtung bei den analytischen Methoden führt zu deutlich längeren Fahnen im Vergleich zu den numerischen Methoden. Bereits geringe Variationen der Fließrichtung würden bei

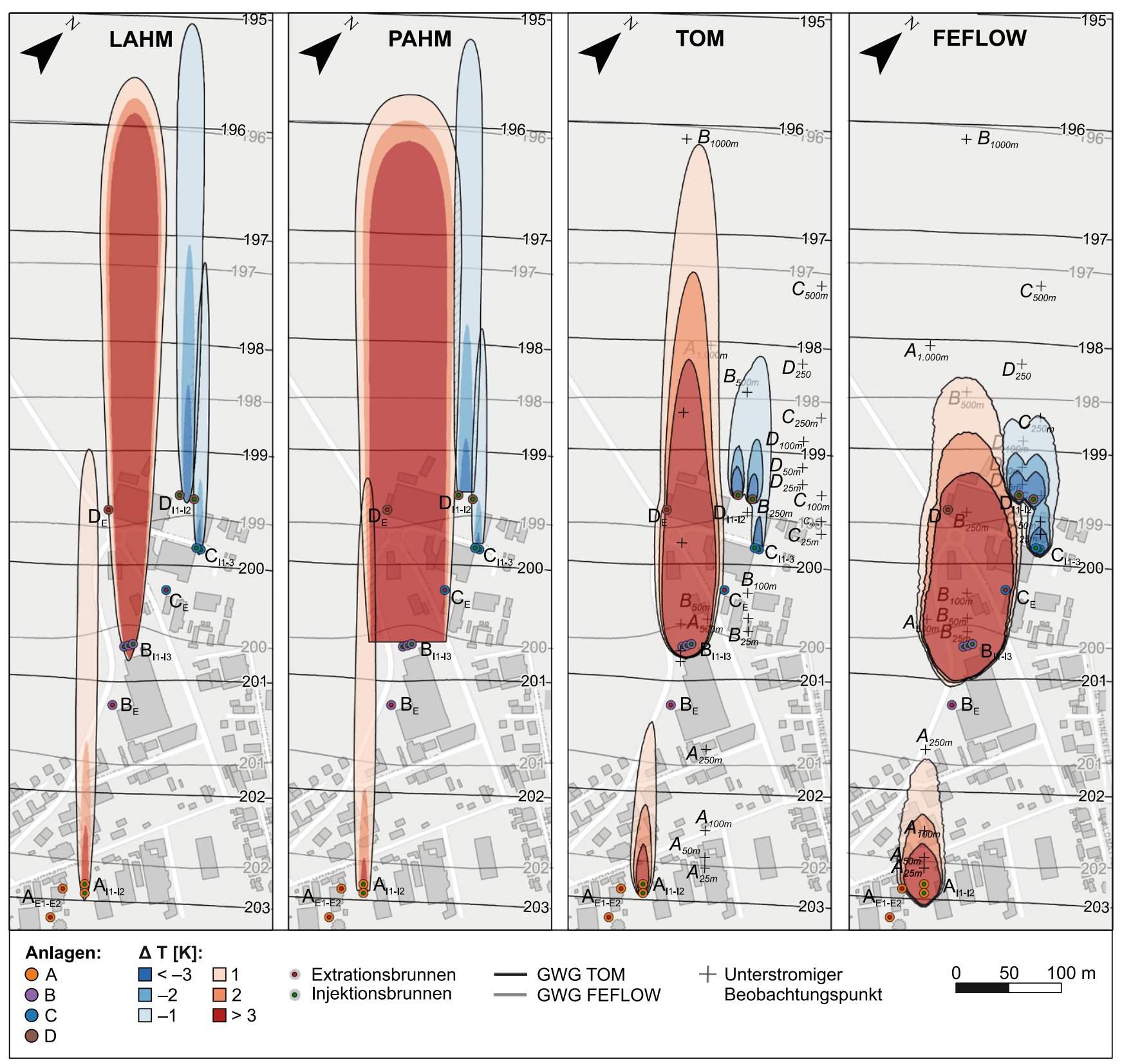

Abb. 4 Vergleich der Temperaturfahnen der analytischen Modelle (LAHM, PAHM), des TOM-Modells und des FEFLOW-Modells. Die TOMund FEFLOW-Fahnen zeigen die maximale Abkühlung und Erwärmung über alle Tiefen im Modell während des Berechnungszeitraums von 5 Jahren

Fig. 4 Comparison of the temperature plumes of the analytical models (LAHM, PAHM), the TOM model and the FEFLOW model. The TOM and FEFLOW plumes show the maximum cooling and warming over all depths in the model during the calculation period. All depths in the model are during a calculation period of 5 years 
beiden analytischen Lösungen zu Überlagerungen mit den Anlagen B-D führen. TOM und FEFLOW liefern hier sehr ähnliche Ergebnisse. TOM erzeugt im Vergleich zu FEFLOW schmalere und etwas längere Temperaturfahnen.

- Anlage B (Kühlung, ganzjährig, $\Delta \mathrm{T}$ : $7 \mathrm{~K}$, 2.123.772kWh/a): Diese Anlage ist für über $90 \%$ des Wärmeenergieeintrags der vier Anlagen verantwortlich. PAHM und LAHM erzeugen jeweils $1 \mathrm{~K}$-Temperaturfahnen von über $500 \mathrm{~m}$ Längsausdehnung. Die laterale Ausbreitung der Temperaturfahnen ist bei der linearen analytischen Lösung (LAHM) deutlich geringer. Die durch TOM bestimmte Temperaturfahne hingegen ist mit einer Länge von $366 \mathrm{~m}$ zwar deutlich kürzer als bei den analytischen Modellen, aber rund $65 \%$ länger und 35\% schmaler als die mit FEFLOW ermittelte Temperaturfahne. Die mit FEFLOW berechneten Temperaturfelder zeigen eine deutliche Tendenz hinsichtlich einer thermisch/hydraulischen Kurzschlussströmung in Richtung des Entnahmebrunnens der Anlage B. Alle vier Modelle zeigen eine Beeinflussung des Entnahmebrunnens der Anlage D von bis zu über $3 \mathrm{~K}$, ausgehend von Anlage B. Bei FEFLOW und PAHM liegt der Entnahmebrunnen der Anlage $\mathrm{C}$ ebenfalls innerhalb des $>3 \mathrm{~K}$ Temperaturfeldes der Anlage B.

- Anlage C (Heizung ganzjährig, $\Delta \mathrm{T}$ : $-4 \mathrm{~K}, 111.767 \mathrm{kWh} / \mathrm{a})$ und Anlage D (kombiniert, Heizung: September-April, Kühlung April-September, $\Delta \mathrm{T}$ : $\pm 4 \mathrm{~K}, 327.892 \mathrm{kWh} / \mathrm{a}$ ): Auch hier entwickeln sich bei den analytischen Lösungen deutlich längere Fahnen, besonders gegenüber der durch FEFLOW simulierten Fahne. TOM erzeugt gegenüber FEFLOW eine rund $35 \%$ längere und $25 \%$ schmalere Fahne.

\section{Vergleich von FEFLOW und TOM}

Wichtigste Faktoren für die Art der Ausbreitung von Temperaturfahnen im Grundwasser sind die Parameter Abstandsgeschwindigkeit, Transmissivität, Dispersion, effektive Wärmekapazität und Wärmeaustausch mit der Atmosphäre. In Grundwassermodellen werden diese EinflussgröBen, je nach Datenlage und Modellstruktur, unterschiedlich stark abstrahiert. Da unter diesen eine Wechselwirkung besteht, ist eine Quantifizierung der Einflüsse der einzelnen Parameter jedoch nur bedingt möglich. Nichtsdestotrotz, wurden in vorherigen Arbeiten Sensitivitätsanalysen zum thermischen Einfluss von Grundwasserwärmepumpen mithilfe von vereinfachten numerischen und analytischen Modellen durchgeführt. Piga et al. (2017) sowie Pophillat et al. 2020 untersuchten den Einfluss von thermischen und hydraulischen Parametern (hydraulische Leitfähigkeit, hydraulischer Gradient, Filtergeschwindigkeit, Porosität, thermische Dispersivität etc.) sowie der Anlagenabbildung (stationär als Jahresmittel vs. instationär) auf die langfristige Entwicklung von Temperaturfahnen. Die Ergebnisse zeigen einen dominierenden Einfluss der Filtergeschwindigkeit (als Produkt der hydraulischen Durchlässigkeit und des hydraulischen Gradienten) sowie der Dispersionskoeffizienten.

Durch die vereinfachte Modellstruktur von TOM beträgt die Transmissivität für alle Brunnenstandorte $3 \cdot 10^{-2} \mathrm{~m}^{2} / \mathrm{s}$ (Abb. 5a). Bei FEFLOW variiert diese im Bereich zwischen $1,5-2,3 \cdot 10^{-2} \mathrm{~m}^{2} / \mathrm{s}$. Die etwas geringeren Werte bei FEFLOW resultieren aus der variablen Schichtmächtigkeit der Neuenburg-Formation und dem hydraulisch wirksamen, geringmächtigen Riegel-Horizont im oberen Abschnitt der Breisgau-Formation. Eine geringere Transmissivität, bedingt durch eine geringere Aquifermächtigkeit, führt zu einer seichteren Ausbreitung und breiteren Temperaturfahnen. Gleichzeitig steigt das Potenzial für hydraulische und thermische Kurzschlüsse. Dies zeigt sich für Anlage B. Eine oberflächennahe Ausbreitung der Temperaturfahne führt aufgrund der größeren Kontaktfläche zu einer vergleichsweise höheren Wechselwirkung mit der Atmosphäre. In Abb. $5 b$ ist der bilanzierte Atmosphärenaustausch für TOM (als Dirichlet-Randbedingung) und FEFLOW (sowohl berechnet als Dirichlet- als auch mit Cauchy-Randbedingungen) für die Gesamtheit aller Anlagen dargestellt. Bei den FEFLOW-Modellen wird insgesamt mehr Wärmeenergie aus dem System ausgetragen (vergleichsweise stärker negative Werte, d.h. es wird industriell erzeugte Abwärme über Konduktion vom Grundwasser an die Atmosphäre abgegeben). Der Energiestrom in die Atmosphäre steigt bei allen Modellen mit Größenzunahme der Warmwasserfahnen. Dies zeigt sich deutlich am Hauptproduzenten der Wärme, Anlage B, dessen Temperaturfahne bei FEFLOW früher einen stationären Zustand erreicht und daher im Vergleich zu der mit TOM ermittelten Fahne deutlich kürzer ausgeprägt ist.

Auch unterschiedliche modellinterne Berechnungsarten, wie die Berechnungen der effektiven Wärmeleitfähigkeit (Abb. 5c, d), können zu Unterschieden in den Simulationsergebnissen führen. Hier werden in TOM, bei gleichen Eingangsparametern, systematisch geringere effektive Wärmeleitfähigkeiten als bei FEFLOW simuliert. Eine hohe Wärmeleitfähigkeit begünstigt den konduktiven Wärmestrom in der ungesättigten Zone, die zum Austausch mit der Atmosphäre führt. Die Modelltopographie von FEFLOW nutzt in dieser Studie das digitale Geländemodell DGM10 (BKG 2020). Bei TOM wird die GOK eines Bezugspunkts für die gesamte Modellfläche verwendet. Dadurch ergeben sich weiterhin lokale Unterschiede des Flurabstandes bzw. des ungesättigten Bereichs und damit des konduktiven Austauschs. 


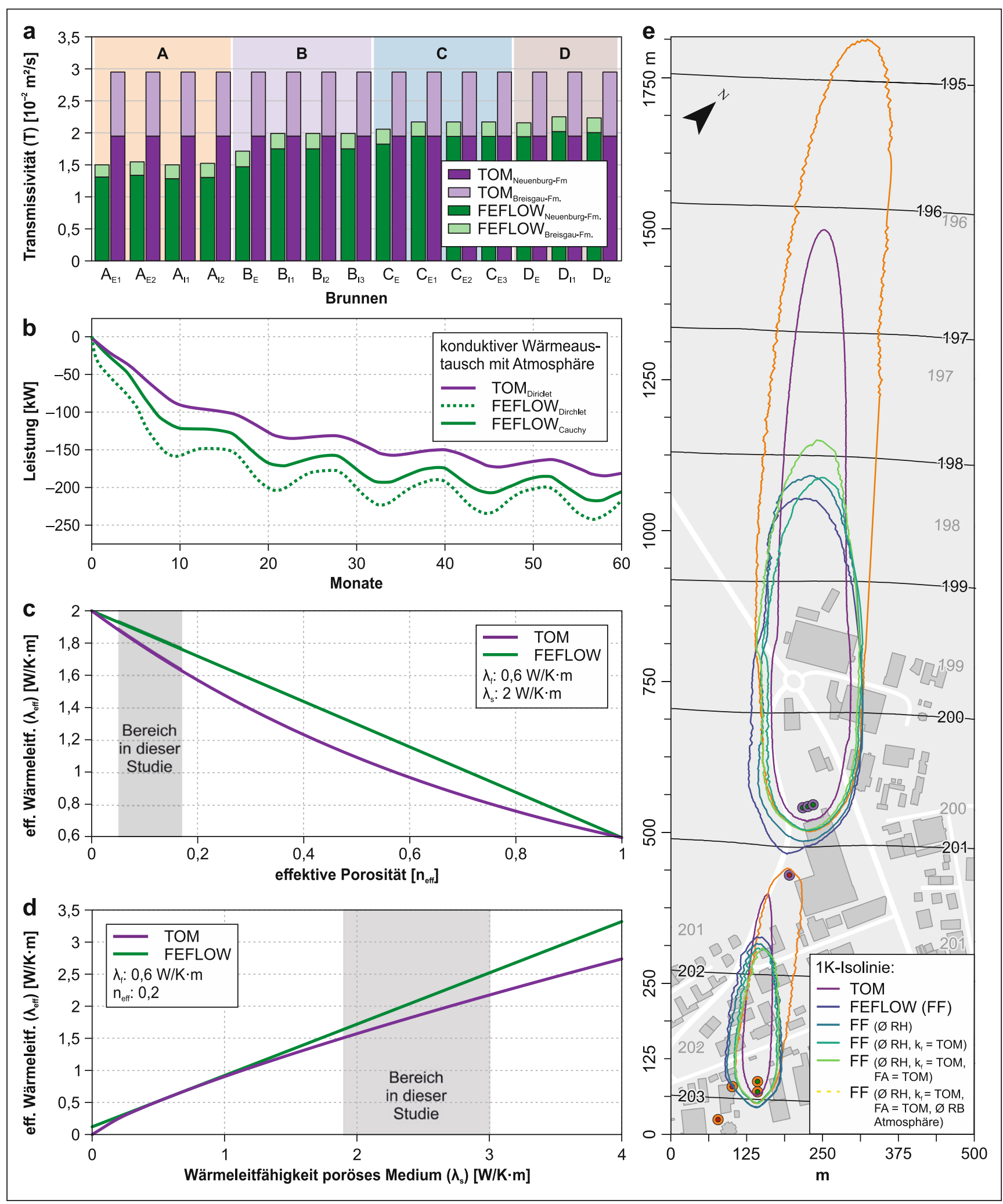

Abb. 5 a Transmissivität der Brunnen bei FEFLOW und TOM; b Bilanzierter Wärmeaustausch mit der Atmosphäre; c Abhängigkeit effektive Wärmeleitfähigkeit von der effektiven Porosität; d Abhängigkeit effektive Wärmeleitfähigkeit von der Wärmeleitfähigkeit; e Maximale Erwärmung über Simulationszeitraum (5 Jahre) Anlage A und B

Fig. 5 a Transmissivity of the wells in FEFLOW and TOM; b Balanced heat exchange with the atmosphere; $\mathbf{c}$ Dependence of effective thermal conductivity on effective porosity; $\mathbf{d}$ Dependence of effective thermal conductivity on thermal conductivity; e Maximum increase in temperature over the simulation period (5 years) for wells $\mathrm{A}$ and $\mathrm{B}$ 

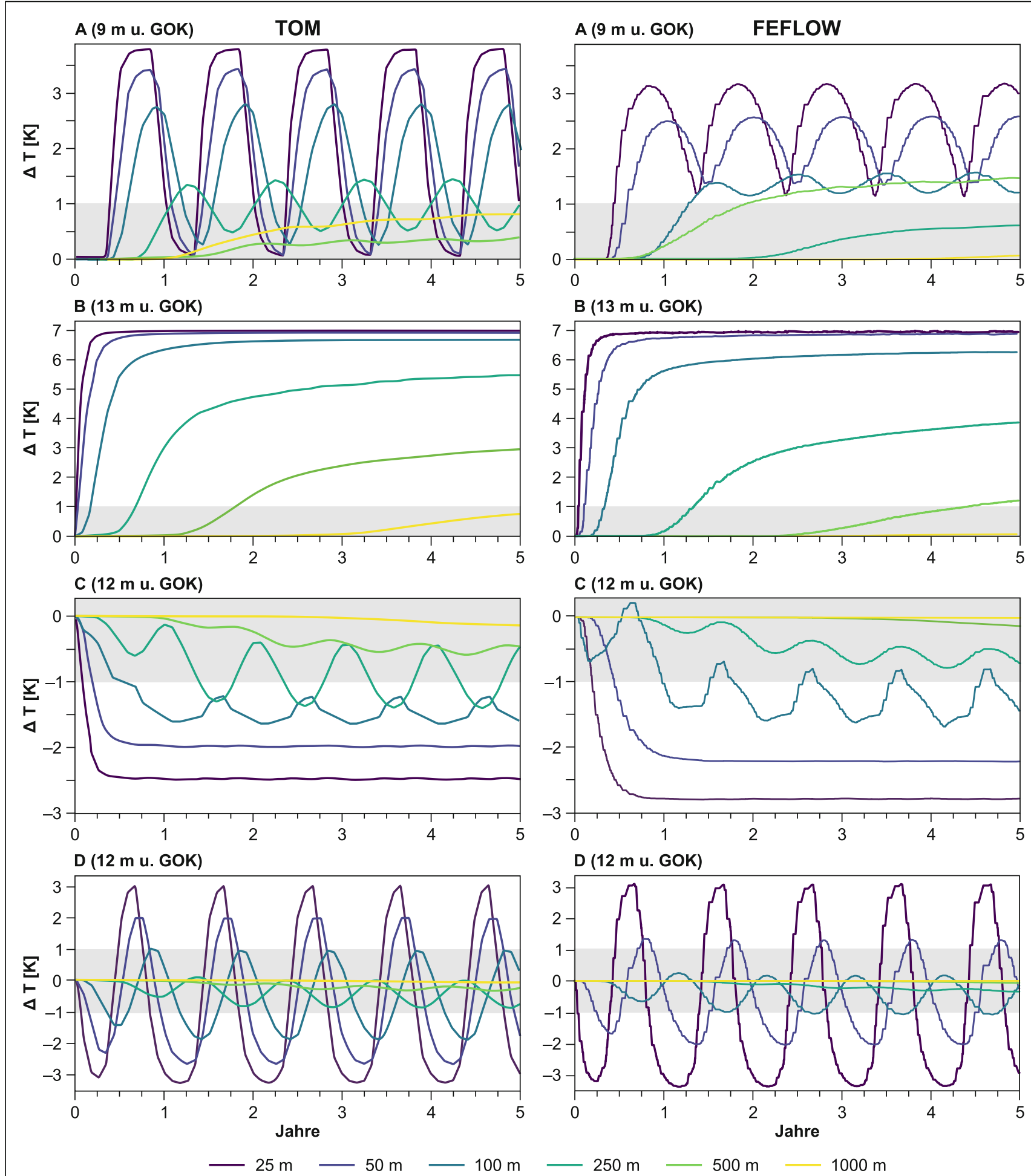

Abb. 6 Modellierte Temperaturprofile mit den Modellen TOM (links) und FEFLOW im Abstrom der Anlagen. Die Lage der Messpunkte ist in Abb. 4a gezeigt (schwarze Kreuze)

Fig. 6 Temperature profiles modelled with TOM (left) and FEFLOW in the downstream of the wells. The location of the measurement points is shown in Fig. 4a (black crosses) 


\section{Quantifizierung der Einflüsse von Modellvereinfachungen}

Abb. 5e zeigt die in TOM und FEFLOW simulierten maximalen Ausbreitungen der $1 \mathrm{~K}$-Isothermen der Anlagen A und B. Um die Auswirkungen einzelner Modellvereinfachungen aufzuzeigen, wurde die Komplexität des FEFLOW Modells schrittweise reduziert (Entfernung des Zwischenhorizonts, homogene $\mathrm{k}_{\mathrm{f}}$-Wert-Verteilung und Flurabstand). Mit Entfernung der hydraulischen Trennschicht (RiegelHorizont) nimmt, aufgrund der Transmissivitätszunahme, die Rückstromrate in Richtung der Entnahmebrunnen der beiden Anlagen A und B ab. Die Fahnenlänge der Anlage B wächst um 7\%, die Fahnenbreite nimmt um 10\% ab. Den stärksten Einfluss zeigt die Veränderung des Flurabstands. Der Wärmestrom durch die ungesättigte Zone in die Atmosphäre wird durch die Erhöhung des Flurabstands reduziert, die Fahne bei Anlage B deutlich länger (17\%). Wie stark der Einfluss des Wärmeaustausches zwischen Boden und Atmosphäre tatsächlich ist, zeigen die orangen $1 \mathrm{~K}$-Fahnen (keine Atmosphärenrandbedingung).

In Abb. 6 ist die zeitliche Temperaturentwicklung im Grundwasserabstrom der Anlagen für die numerischen Modelle TOM und FEFLOW gegenübergestellt. Die Abbildungen geben die zeitliche und räumliche Temperaturfahnenentwicklung im Abstrom der Schluckbrunnen in den Entfernungen $25 \mathrm{~m}, 50 \mathrm{~m}, 100 \mathrm{~m}, 250 \mathrm{~m}, 500 \mathrm{~m}$ und $1000 \mathrm{~m}$ in der Tiefe der Filtermitte der Schluckbrunnen wieder. Die Lagen der Punkte sind zusätzlich in Abb. 4 als schwarze Kreuze dargestellt. Die resultierenden Temperaturberechnungen der Simulationen mit FEFLOW und TOM zeigen ähnliche saisonale Temperaturverläufe, Amplituden und Trends. Temperaturunterschiede zwischen FEFLOW und TOM an den gleichen Punkten liegen meist unter 0,5 K. Der leichte zeitliche Versatz der Zeitreihen von FEFLOW und TOM ist auf den oben genannten unterschiedlichen Modellaufbau, die unterschiedliche Parametrisierung und die zeitliche Diskretisierung sowie auf lokale Interaktion mit Anlagen im Abstrom zurückzuführen. Die Temperaturzunahme in $1000 \mathrm{~m}$ Entfernung zur Anlage A ist auf den Einfluss der Anlage B zurückzuführen.

\section{Fazit}

Diese Studie zeigt, welchen Einfluss die Wahl des Berechnungsmodells und der Modell-komplexität auf die Simulationsergebnisse von Temperaturfeldberechnungen für Grundwasserwärmepumpenanlagen unter den Mindestanforderungen des Leitfadens zur Nutzung von GWWP in Baden-Württemberg haben. Es wurde der Anlagenbetrieb von vier industriellen Großanlagen jeweils mit zwei analytischen Modellen (LAHM, PAHM), einem Prinzipmodell
(TOM) und einem Planungsmodell (FEFLOW) simuliert, und anschließend wurden die Ergebnisse verglichen.

Beide analytischen Modelle erzeugen deutlich längere und lateral unterschiedlich ausgedehnte Temperaturfahnen im Vergleich zu den numerischen Berechnungen. Gründe für diese Überschätzung bei den analytischen Lösungen sind der stationäre Anlagenbetrieb, die Vernachlässigung des vertikalen Wärmeaustauschs mit der Atmosphäre und dem Liegenden (Pophillat et al. 2018) sowie die Nichtberücksichtigung einer möglichen Interferenz mit anderen Anlagen.

Die Unterschiede der Temperaturfahnen von Prinzipmodell (TOM) und Planungsmodell (FEFLOW) sind hauptsächlich auf die bei TOM getroffenen Vereinfachungen, wie homogene Parametrisierung, reduzierter Schichtaufbau und deren Wechselwirkungen zurückzuführen. Vor allem der in den beiden Modellen implementierte unterschiedliche Wärmeaustausch mit der Atmosphäre hat einen großen Einfluss auf die laterale Ausbreitung der Temperaturfahne und führt zu kürzeren Fahnen bei FEFLOW. Mit zunehmender Kontaktfläche mit der ungesättigten Zone bei großen Fahnen tritt dieser Effekt zunehmend in den Vordergrund, weswegen ab einer Förderrate von ca. $\geq 101 /$ s (im Jahresdurchschnitt) einem detaillierten Bewirtschaftungsmodell grundsätzlich der Vorzug zu geben ist.

Der direkte Vergleich zwischen TOM und FEFLOW ist nur bedingt möglich, da einerseits die unterschiedlichen Berechnungen der Wärmeleitfähigkeiten und andererseits die in FEFLOW zweifellos genauere Abbildung der Flurabstände Unterschiede in den Ergebnissen verursachen. Zwar zeigt ein Vergleich der Ergebnisse, zu welchen Abweichungen diese Unterschiede im Simulationsprozess führen, aber da letztlich die ,realen Verhältnisse" als Referenz nicht bekannt sind, bleibt eine Bewertung schwierig.

Der Vergleich der Simulationsergebnisse für eine Fallstudie im Porengrundwasserleiter eines Gewerbegebiets in der Freiburger Bucht hat gezeigt, dass das Thermische Online-Modell TOM als Prinzipmodell den verwendeten analytischen Modellen deutlich überlegen ist und für Anlagen $<101 /$ s vergleichbare Ergebnisse zu dem wesentlich komplexeren und rechenintensiveren FEFLOW-Modell liefert. Für die meisten Anwendungsfälle der oberflächennahen Geothermie (kleinere bis mittlere GWWP-Anlagen, homogene $\mathrm{k}_{\mathrm{f}}$-Wert-Verteilung) bietet TOM gegenüber den vereinfachten analytischen Modellen daher einen Mehrwert an Vorhersagegenauigkeit.

Dies macht TOM zu einem praktischen und anwenderfreundlichen Werkzeug, das den wasserrechtlichen Genehmigungsprozess von kleineren bis mittleren Anlagen vereinfachen, standardisieren und transparenter machen kann. Allerdings ersetzt TOM kein detailliertes Wärmetransportmodell mit komplexen Randbedingungen wie beispielsweise jahreszeitliche Veränderungen, Vorfluter in 
nächster Umgebung der betrachteten Anlage oder thermische Austauschprozesse in urbanen Räumen. Ein komplexer Schichtaufbau, eine heterogene $\mathrm{k}_{\mathrm{f}}$-Wert-Verteilung und Wechselwirkung mit Oberflächengewässern können damit nicht abgebildet werden. Durch den Aufbau eines hydrogeologischen Konzeptmodells muss beurteilt werden, ob die bei TOM getroffenen Vereinfachungen für die vorliegenden Begebenheiten zulässig und akzeptabel sind oder gegebenenfalls durch ein kalibriertes Detailmodell ergänzt werden müssen. Weiterhin sollten Variationsrechnungen durchgeführt werden, um die lokale Variabilität der Parameter abzuschätzen.

Sehr große Anlagen mit Förderraten von $>101 /$ s erfordern aufgrund der möglichen Auswirkungen einen wesentlich größeren Untersuchungs- und Modellierungsaufwand und daher den Aufbau eines Planungs- oder Bewirtschaftungsmodells gemäß DVGW W107 (A).

Danksagung Für die Unterstützung bei der Modellentwicklung des Thermischen Online-Modells (TOM) bedanken wir uns bei Bernhard Keim von der Ingenieurgesellschaft Prof. Kobus und Partner GmbH und Bernd Flemisch von der Universität Stuttgart. Ein weiterer Dank geht an Anja Hollerbach vom Landratsamt Karlsruhe, Amt für Umwelt und Arbeitsschutz und Thomas Gudera von der Landesanstalt für Umwelt Baden Württemberg (LUBW) für die Unterstützung dieser Arbeit. Für die konstruktiven Kommentare der beiden Gutachter möchten wir uns ebenfalls bedanken.

Funding Open Access funding enabled and organized by Projekt DEAL.

Open Access Dieser Artikel wird unter der Creative Commons Namensnennung 4.0 International Lizenz veröffentlicht, welche die Nutzung, Vervielfältigung, Bearbeitung, Verbreitung und Wiedergabe in jeglichem Medium und Format erlaubt, sofern Sie den/die ursprünglichen Autor(en) und die Quelle ordnungsgemäß nennen, einen Link zur Creative Commons Lizenz beifügen und angeben, ob Änderungen vorgenommen wurden.

Die in diesem Artikel enthaltenen Bilder und sonstiges Drittmaterial unterliegen ebenfalls der genannten Creative Commons Lizenz, sofern sich aus der Abbildungslegende nichts anderes ergibt. Sofern das betreffende Material nicht unter der genannten Creative Commons Lizenz steht und die betreffende Handlung nicht nach gesetzlichen Vorschriften erlaubt ist, ist für die oben aufgeführten Weiterverwendungen des Materials die Einwilligung des jeweiligen Rechteinhabers einzuholen.

Weitere Details zur Lizenz entnehmen Sie bitte der Lizenzinformation auf http://creativecommons.org/licenses/by/4.0/deed.de.

\section{Literatur}

Abu-Hamdeh, N.H.: Thermal properties of soils as affected by density and water content. Biosyst. Eng. 86, 97-102 (2003). https://doi. org/10.1016/S1537-5110(03)00112-0

Attard, G., Rossier, Y., Winiarski, T., Eisenlohr, L.: Deterministic modeling of the impact of underground structures on urban groundwater temperature. Sci. Total Environ. 572, 986-994 (2016). https://doi.org/10.1016/j.scitotenv.2016.07.229

Bear, J.: Dynamics of Fluids in Porous Media. Dover, New York (1972)

Becker, D., Epting, J.: Thermischer Einfluss urbaner Untergrundstrukturen auf die Grundwassertemperaturen im Kanton Basel-Stadt.
Grundwasser 26(3), 269-288 (2021). https://doi.org/10.1007/ s00767-021-00483-1

BKG: Digitales Geländemodell 10 (DGM10). Bundesamt für Kartographie und Geodäsie, (2020)

Blum, P., Menberg, K., Koch, F., Benz, S.A., Tissen, C., Hemmerle, H., Bayer, P.: Is thermal use of groundwater a pollution? J. Contam. Hydrol. (2021). https://doi.org/10.1016/j.jconhyd.2021.103791

Boettcher, F., Casasso, A., Götzl, G., Zosseder, K.: TAP-thermal aquifer potential: a quantitative method to assess the spatial potential for the thermal use of groundwater. Renew. Energy 142, 85-95 (2019). https://doi.org/10.1016/j.renene.2019.04.086

Bucci, A., Barbero, D., Lasagna, M., Forno, M.G., De Luca, D.A.: Shallow groundwater temperature in the Turin area (NW Italy): vertical distribution and anthropogenic effects. Environ. Earth Sci. 76, 221 (2017). https://doi.org/10.1007/s12665-017-6546-4

Dalla Santa, G., Peron, F., Galgaro, A., Cultrera, M., Bertermann, D., Mueller, J., Bernardi, A.: Laboratory measurements of gravel thermal conductivity: an update methodological approach. Energy Procedia 125, 671-677 (2017). https://doi.org/10.1016/j. egypro.2017.08.287

De Marsily, G.: Quantitative Hydrogeology: Groundwater Hydrogeology for Engineers. Academic Press, London (1980)

Diersch, H.-J.G.: FEFLOW. Springer, Berlin, Heidelberg (2014) https://doi.org/10.1007/978-3-642-38739-5

DIN e.V.: DIN 18130-1: Bestimmung des Wasserdurchlässigkeitsbeiwerts - Teil 1: Laborversuche. DIN e.V., Berlin (1998)

Domenico, P.A., Robbins, G.A.: A new method of contaminant plume analysis. Ground Water (1985). https://doi.org/10.1111/j.17456584.1985.tb01497.x

DVGW: W 107 (a): Technische Regel - Arbeitsblatt 2016-02: Aufbau und Anwendung numerischer Grundwassermodelle in Wassergewinnungsgebieten (A). Deutscher Verein des Gas- und Wasserfaches e.V., Bonn (2016)

Epting, J., Böttcher, F., Mueller, M.H., García-Gil, A., Huggenberger, P., Vázquez-Suñe, E.: Development of concepts for the management of thermal resources in urban areas-Assessment of transferability from the Basel (Switzerland) and Zaragoza (Spain) case studies. J. Hydrol. 548(19), 697-715 (2017). https://doi.org/10. 1016/j.jhydrol.2017.03.057

Epting, J., García-Gil, A., Huggenberger, P., Vázquez-Suñe, E., Mueller, M.H.: City-scale solutions for the energy use of shallow urban subsurface resources-Bridging the gap between theoretical and technical potentials. Renew. Energy 147(1), 751-763 (2019). https://doi.org/10.1016/j.renene.2019.09.021

Flemisch, B., Darcis, M., Erbertseder, K., Faigle, B., Lauser, A., Mosthaf, K., Müthning, S., Nuske, P., Tatomir, A., Wolff, M., Helmig, R.: DuMux: DUNE for multi-\{phase, component, scale, physics, ... flow and transport in porous media. Adv. Water Resour. 34(9), 1102-1112 (2011). https://doi.org/10.1016/j.advwatres. 2011.03.007

Frey, C.: Grundwasserwärmenutzung (Umkirch). Unveröffentlichtes Gutachten. Freiburg (2016)

Fry, V.A.: Lessons from London: regulation of open-loop ground source heat pumps in central London. Q. J. Eng. Geol. Hydrogeol. 42, 325-334 (2009). https://doi.org/10.1144/1470-9236/08087

García-Gil, A., Vázquez-Suñe, E., Alcaraz, M.M., Juan, A.S., Sánchez-Navarro, J.Á., Montlleó, M., Rodríguez, G., Lao, J.: GISsupported mapping of low-temperature geothermal potential taking groundwater flow into account. Renew. Energy 77, 268-278 (2015). https://doi.org/10.1016/j.renene.2014.11.096

Garling, F., Dittrich, J.: Hydrogeologie - Gesteinsbemusterung. VEB Deutscher Verlag für Grundstoffindustrie (1979)

Haehnlein, S., Bayer, P., Blum, P.: International legal status of the use of shallow geothermal energy. Renew. Sustain. Energy Rev. 14, 2611-2625 (2010a). https://doi.org/10.1016/j.rser.2010.07.069

Häfner, F., Sames, D., Voigt, H.-D.: Wärme- und Stofftransport, Mathematische Methoden. Springer, Berlin (1992) 
Hähnlein, S., Molina-Giraldo, N., Blum, P., Bayer, P., Grathwohl, P.: Ausbreitung von Kältefahnen im Grundwasser bei Erdwärmesonden. Grundwasser 15(2), 123-133 (2010b). https://doi.org/10. 1007/s00767-009-0125-x

Hähnlein, S., Bayer, P., Ferguson, G., Blum, P.: Sustainability and policy for the thermal use of shallow geothermal energy. Energy Policy 59, 914-925 (2013). https://doi.org/10.1016/j.enpol.2013.04. 040

Hecht-Méndez, J., Molina-Giraldo, N., Blum, P., Bayer, P.: Evaluating MT3DMS for heat transport simulation of closed shallow geothermal systems. Ground Water 48(5), 741-756 (2010). https:// doi.org/10.1111/j.1745-6584.2010.00678.x

Kinzelbach, W.: Numerische Methoden zur Modellierung des Transports von Schadstoffen im Grundwasser. Schriftenreihe gwf Wasser Abwasser. R. Oldenbourg, München-Wien (1987)

Kobus und Partner: Manual für das Thermische Online-Modell (TOM) Bd. 60. (2020)

Koch, T., Gläser, D., Weishaupt, K., Ackermann, S., Beck, M., Becker, B., Burbulla, S., Class, H., Coltman, E., Emmert, S., Fetzer, T., Grüninger, C., Heck, K., Hommel, J., Kurz, T., Lipp, M., Mohammadi, F., Scherrer, S., Schneider, M., Seitz, G., Stadler, L., Utz, M., Weinhardt, F., Flemisch, B.: DuMux 3-an open-source simulator for solving flow and transport problems in porous media with a focus on model coupling. Comput. Math. Appl. (2020). https://doi.org/10.1016/j.camwa.2020.02.012

LGRB: Hydrogeologischer Bau und Aquifereigenschaften der Lockergesteine im Oberrheingraben (Baden-Württemberg). LGRB Informationen, Bd. 19. Regierungspräsidium Freiburg, Abteilung 9 Landesamt für Geologie, Rohstoffe und Bergbau (LGRB), (2007)

Lo Russo, S., Gnavi, L., Roccia, E., Taddia, G., Verda, V.: Groundwater Heat Pump (GWHP) system modeling and Thermal Affected Zone (TAZ) prediction reliability: Influence of temporal variations in flow discharge and injection temperature. Geothermics 51, 103-112 (2014). https://doi.org/10.1016/j.geothermics.2013. 10.008

LUBW: Hydrogeologischer Bau und hydraulische Eigenschaften INTERREG III A-Projekt MoNit „Modellierung der Grundwasserbelastung durch Nitrat im Oberrheingraben“. Landesanstalt für Umwelt, Baden-Württemberg (2006)

Marotz, G.: Technische Grundlagen einer Wasserspeicherung im natürlichen Untergrund. Schriftenreihe KWK, Bd. 18. Wasser u. Boden, Hamburg (1968)

Menberg, K., Steger, H., Zorn, R., Reuß, M., Pröll, M., Bayer, P., Blum, P.: Bestimmung der Wärmeleitfähigkeit im Untergrund durch Labor- und Feldversuche und anhand theoretischer Modelle. Grundwasser 18(2), 103-116 (2013). https://doi.org/10.1007/ s00767-012-0217-x

Mueller, M.H., Huggenberger, P., Epting, J.: Combining monitoring and modelling tools as a basis for city-scale concepts for a sustainable thermal management of urban groundwater resources. Sci. Total Environ. 627, 1121-1136 (2018). https://doi.org/10.1016/j. scitotenv.2018.01.250

Márquez, J.M.A., Bohórquez, M.Á.M., Melgar, S.G.: Ground thermal diffusivity calculation by direct soil temperature measurement. application to very low enthalpy geothermal energy systems. Sensors 16, 9-13 (2016). https://doi.org/10.3390/s16030306

Piga, B., Casasso, A., Pace, F., Godio, A., Sethi, R.: Thermal impact assessment of groundwater heat pumps (GWHPs): rigorous vs. simplified models. Energies 10, 1385 (2017). https://doi.org/10. 3390/en10091385

Pophillat, W., Attard, G., Bayer, P., Hecht-Méndez, J., Blum, P.: Analytical solutions for predicting thermal plumes of groundwater heat pump systems. Renew. Energy 147, 2696-2707 (2018). https:// doi.org/10.1016/j.renene.2018.07.148

Pophillat, W., Bayer, P., Teyssier, E., Blum, P., Attard, G.: Impact of groundwater heat pump systems on subsurface temperature under variable advection, conduction and dispersion. Geothermics (2020). https://doi.org/10.1016/j.geothermics.2019.101721

Poppei, J., Schwarz, R.: A simple tool for designing and assessing thermal groundwater utilization. In: PROCEEDINGS, Thirty-Sixth Workshop on Geothermal Reservoir Engineering. Stanford University, Stanford, California (2011)

Rivera, J.A., Blum, P., Bayer, P.: A finite line source model with Cauchy-type top boundary conditions for simulating near surface effects on borehole heat exchangers. Energy 98, 50-63 (2016). https://doi.org/10.1016/j.energy.2015.12.129

RVSO: Mittelwasserstand-Grundwassergleichenplan (MW 1986). Regionalverband südlicher Oberrhein (RVSO). Bereitgestellt durch Landratsamt Breisgau-Hochschwarzwald (1986)

Sauty, J.-P.: An analysis of hydrodispersive transfer in aquifers. Water Resour. Res. 16(1), 145-158 (1980). https://doi.org/10.1029/ WR016i001p00145

Stathers, R.J., Spittlehouse, D.L.: Forest Soil Temperature Manual (1990)

Stauffer, F., Bayer, P., Blum, P., Molina-Giraldo, N.: Thermal Use of Shallow Groundwater. Taylor \& Francis, (2014). Reprint Edition

Steiner, C., Heimlich, K., Hilberg, S.: Vergleichende Temperaturfahnenprognose anhand zweier industriell genutzter Grundwasserwärmepumpen: FEFLOW vs. ÖWAV-Modell. Grundwasser (2016). https://doi.org/10.1007/s00767-016-0328-x

Tissen, C., Menberg, K., Bayer, P., Blum, P.: Meeting the demand: geothermal heat supply rates for an urban quarter in Germany. Geotherm. Energy (2019). https://doi.org/10.1186/s40517-019-01258

Tsagarakis, K.P., Efthymiou, L., Michopoulos, A., Mavragani, A., Anđelković, A.S., Antolini, F., Bacic, M., Bajare, D., Baralis, M., Bogusz, W., Burlon, S., Figueira, J., Genç, M.S., Javed, S., Jurelionis, A., Koca, K., Ryżyński, G., Urchueguia, J.F., Žlender, B.: A review of the legal framework in shallow geothermal energy in selected European countries: need for guidelines. Renew. Energy 147, 2556-2571 (2020). https://doi.org/10.1016/j.renene.2018. 10.007

UM BW: Weitergehende Beschreibung des Gefährdeten Grundwasserkörpers 16.5 „Ortenau-Ried“. Umweltministerium, Baden-Württemberg (2004)

UM BW: Leitfaden zur Nutzung von Erdwärme mit Grundwasserwärmepumpen. Umweltministerium, Baden-Württemberg (2009a)

UM BW: Arbeitshilfe zum Leitfaden zur Nutzung von Erdwärme mit Grundwasserwärmepumpen. Umweltministerium, BadenWürttemberg (2009b)

VDE: Potenziale für Strom im Wärmemarkt bis 2050 (VDE Studie). Verband der Elektrotechnik Elektronik Informationstechnik e. V., Frankfurt am Main (2015)

VDI: 4640, Blatt 1: Thermische Nutzung des Untergrunds - Grundlagen, Genehmigungen, Umweltaspekte. Verein Deutscher Ingenieure, Berlin (2010)

Wagner, V., Bayer, P., Kübert, M., Blum, P.: Numerical sensitivity study of thermal response tests. Renew. Energy 41, 245-253 (2012). https://doi.org/10.1016/j.renene.2011.11.001

Hinweis des Verlags Der Verlag bleibt in Hinblick auf geografische Zuordnungen und Gebietsbezeichnungen in veröffentlichten Karten und Institutsadressen neutral. 